Draft VERSION MARCH 12, 2021

Typeset using LATEX preprint2 style in AASTeX63

\title{
A search for cloud cores affected by shocked carbon chain chemistry in L1251
}

X.-C. Liu (刘训川), ${ }^{1,2}$ Y. Wu, ${ }^{1,2}$ C. Zhang (张超), ${ }^{1,3}$ X. Chen, ${ }^{4}$ L.-H. Lin,,${ }^{5,6,7}$ S.-L. Qin, ${ }^{3}$ T. Liu, ${ }^{8}$ C. HenKel, ${ }^{9,}, 10$

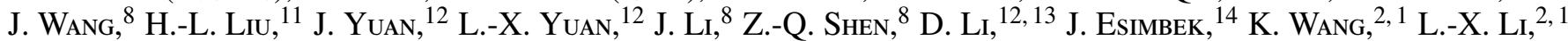
Kee-Tae Kim, ${ }^{15,16}$ L. Zhu, ${ }^{12}$ D. Madones, ${ }^{17}$ N. Inostroza,,${ }^{18}$ F.-Y. Meng, ${ }^{19}$ T. Zhang, ${ }^{19}$ K. Tatematsu, ${ }^{20}$ Y. Xu, ${ }^{21}$ B.-G. Ju, ${ }^{21}$ A. Kraus ${ }^{13}$ and F.-W. Xu ${ }^{1,2}$

${ }^{1}$ Department of Astronomy, School of Physics, Peking University, 100871 Beijing, China

${ }^{2}$ Kavli Institute for Astronomy and Astrophysics, Peking University, 100871 Beijing, China

${ }^{3}$ Department of Astronomy, Yunnan University, Kunming, 650091, China

${ }^{4}$ Center for Astrophysics, GuangZhou University, Guangzhou 510006, China

${ }^{5}$ Purple Mountain Observatory, Chinese Academy of Sciences, 10 Yuanhua Road, Nanjing 210033, P.R. China

${ }^{6}$ Key Laboratory of Radio Astronomy, Chinese Academy of Sciences, 10 Yuanhua Road, Nanjing 210033, P.R. China

${ }^{7}$ School of Astronomy and Space Science, University of Science and Technology of China, 96 Jinzhai Road, Hefei 230026, P.R. China

${ }^{8}$ Shanghai Astronomical Observatory, Chinese Academy of Sciences, Shanghai 200030, China

${ }^{9}$ Max-Planck Institut für Radioastronomie, Auf dem Hügel 69, 53121 Bonn, Germany

${ }^{10}$ Astron. Dept., King Abdulaziz University, P.O. Box 80203, Jeddah 21589, Saudi Arabia

${ }^{11}$ Departamento de Astronomía, Universidad de Concepción, Av. Esteban Iturra s/n, Distrito Universitario, 160-C, Chile

${ }^{12}$ National Astronomical Observatories, Chinese Academy of Sciences, Beijing 100101, China

${ }^{13}$ NAOC-UKZN Computational Astrophysics Centre, University of KwaZulu-Natal, Durban 4000, South Africa

${ }^{14}$ Xinjiang Astronomical Observatory, Chinese Academy of Sciences, 830011, Urumqi, China

${ }^{15}$ Korea Astronomy and Space Science Institute, 776 Daedeokdae-ro, Yuseong-gu, Daejeon 34055, Republic of Korea

${ }^{16}$ University of Science and Technology, Korea (UST), 217 Gajeong-ro, Yuseong-gu, Daejeon 34113, Republic of Korea

${ }^{17}$ Department of Astronomy, University of Chile, Casilla 36-D, Santiago, Chile

${ }^{18}$ Núcleo de Astroquímicay Astrofísica, Instituto de Ciencias Químicas Aplicadas, Facultad de Ingeniería, Universidad Autónoma de Chile Av.

${ }^{19}$ I. Physikalisches Institut, Universität zü Köln, Zülpicher Str. 77, D-50937 Köln, Germany

${ }^{20}$ Nobeyama Radio Observatory, National Astronomical Observatory of Japan, National Institutes of Natural Sciences, $462-2$ Nobeyama, Minamimaki, Minamisaku, Nagano 384-1305, Japan

${ }^{21}$ Purple Mountain Observatory, Qinghai Station, 817000, Delingha, China

\begin{abstract}
We searched for shocked carbon chain chemistry (SCCC) sources with $\mathrm{C}_{3} \mathrm{~S}$ abundances surpassing those of $\mathrm{HC}_{5} \mathrm{~N}$ towards the dark cloud L1251, using the Effelsberg telescope at K-band (18 - 26 GHz). L1251-1 and L1251-3 are identified as the most promising SCCC sources. The two sources harbor young stellar objects. We conducted mapping observations towards L1251-A, the western tail of L1251, at $\lambda \sim 3 \mathrm{~mm}$ with the PMO $13.7 \mathrm{~m}$ and the NRO $45 \mathrm{~m}$ telescopes in lines of $\mathrm{C}_{2} \mathrm{H}, \mathrm{N}_{2} \mathrm{H}^{+}, \mathrm{CS}, \mathrm{HCO}^{+}, \mathrm{SO}, \mathrm{HC}_{3} \mathrm{~N}$ and $\mathrm{C}^{18} \mathrm{O}$ as well as in $\mathrm{CO}$ 3-2 using the JCMT. The spectral data were combined with archival data including Spitzer
\end{abstract}

ywu@pku.edu.cn

1501110219@pku.edu.cn 
and Herschel continuum maps for further analysis. Filamentary sub-structures labeled as F1 to F6 were extracted in L1251, with F1 being associated with L1251-A hosting L1251-1. The peak positions of dense gas traced by $\mathrm{HCO}^{+}$are misaligned relative to those of the dust clumps. Episodic outflows are common in this region. The twisted morphology of F1 and velocity distribution along L1251-A may originate from stellar feedback. SCCC in L1251-1 may have been caused by outflow activities originated from the infrared source IRS1. The signposts of ongoing SCCC and the broadened line widths of $\mathrm{C}_{3} \mathrm{~S}$ and $\mathrm{C}_{4} \mathrm{H}$ in L1251-1 as well as the distribution of $\mathrm{HC}_{3} \mathrm{~N}$ are also related to outflow activities in this region. L1251-1 (IRS1) together with the previously identified SCCC source IRS3 demonstrate that L1251-A is an excellent region to study shocked carbon chain chemistry.

Keywords: ISM: molecules - ISM: abundances - ISM: kinematics and dynamics - ISM: clouds - stars: formation - ISM: Radio lines

\section{INTRODUCTION}

Carbon chain molecules (CCMs) are particularly interesting since they are important tracers of organic interstellar chemistry. The nitrogen-bearing CCMs $\mathrm{HC}_{3} \mathrm{~N} J=1-0$ and $\mathrm{HC}_{5} \mathrm{~N} J=4-3$ were firstly detected towards Sgr B2 in the seventies (Turner 1971; Morris et al. 1976). Soon after, $\mathrm{HC}_{7} \mathrm{~N}$ and $\mathrm{HC}_{9} \mathrm{~N}$ were found in TMC-1 (Kroto et al. 1978; Broten et al. 1978). They are important players in hydrocarbon chemistry (Dickens et al. 2001) and constrain evolutionary stages of dark cold cores (Benedettini et al. 2012). These molecular species can also trace dynamical motions in molecular clouds, including material infall (Friesen et al. 2013). In some cold clouds cyanopolyynes $\left(\mathrm{HC}_{2 n+1} \mathrm{~N}\right)$ are particularly prominent and even $\mathrm{HC}_{11} \mathrm{~N}$ has been detected in TMC1. (Loomis et al. 2020). In star forming regions, cyanopolyynes are usually suppressed, although they can be abundant in some massive star forming regions (Taniguchi et al. 2019).

Another important kind of unsaturated CCMs, sulfur-bearing molecules including $\mathrm{C}_{2} \mathrm{~S}$ and $\mathrm{C}_{3} \mathrm{~S}$, can be used to investigate physical conditions of their production regions. In early cold cores, the column densities of sulfur-bearing molecules are usually positively correlated with those of nitrogen-containing molecules (Suzuki et al. 1992; Hirahara et al. 1992; Fuente et al. 2016). In embed- ded low-mass protostars, significant positive correlations between the cyanopolyynes $\left(\mathrm{HC}_{\mathrm{n}} \mathrm{N}\right)$ and pure hydrocarbon chains $\left(\mathrm{C}_{\mathrm{n}} \mathrm{H}\right)$ were found (Law et al. 2018). C2S lines were not or only marginally detected in a number of low mass star formation cores (Suzuki et al. 1992).

In L1527 (IRAS 04368+2557), Sakai et al. (2008a,b) found strong emissions of highextinction lines $\left(E_{\mathrm{u}}>20 \mathrm{~K}\right)$ of unsaturated hydrocarbons, including $\mathrm{C}_{4} \mathrm{H}_{2}, \mathrm{C}_{4} \mathrm{H}$ and $l-\mathrm{C}_{3} \mathrm{H}_{2}$. A mechanism named warm carbon chain chemistry (WCCC) was put forward to explain the phenomena. In the picture of WCCC, prestellar objects contract and heat the surrounding dust, and the evaporated $\mathrm{CH}_{4}$ reacts with $\mathrm{C}^{+}$to produce $\mathrm{CCMs}$ (Sakai et al. 2008b, 2009). The second WCCC source (I15398-3359) was found in Lupus I (Sakai et al. 2009). Molecular outflows were found in both WCCC sources (Sakai et al. 2008b; Oya et al. 2014; Y1ldiz et al. 2015). The excitation temperatures of hydrocarbons such as $\mathrm{C}_{4} \mathrm{H}_{2}$ and $\mathrm{CH}_{3} \mathrm{CCH}$ are determined to be about $12 \mathrm{~K}$ in both WCCC sources (Sakai et al. 2008a, 2009), which are lower than the sublimation temperature of $\mathrm{CH}_{4}(25 \mathrm{~K})$ but much higher than the excitation temperature of hydrocarbons in TMC-1 (3.8-6.7 K for $\mathrm{C}_{4} \mathrm{H}_{2}$; Kaifu et al. 2004; Sakai et al. 2008b).

Recently we have observed six outflows and five starless Lupus cores using the Shanghai 65 m Tian Ma Radio Telescope (TMRT) to explore 
the production region and excitation mechanism of CCMs (Wu et al. 2019a). All the targets are located within $330 \mathrm{pc}$ from the Earth. The $\mathrm{HC}_{3} \mathrm{~N}$ $J=2-1, \mathrm{HC}_{5} \mathrm{~N} J=6-5, \mathrm{HC}_{7} \mathrm{~N} J=14-13,15-$ $14,16-15$ and $\mathrm{C}_{3} \mathrm{~S} J=3-2$ transitions were observed. These lines were detected towards ten of the eleven observed sources. Three sources, IRAS 20582+7724 (I20582), L1221 and L1251A (corresponding to IRS3 in the L1251-A region, hereafter L1251-IRS3), were explained in terms of a new chemical mechanism - shocked carbon chain chemistry (SCCC); another outflow source, the eastern molecular core of the outflow source L1489 (L1489 EMC) was identified as a particular carbon-chain production region and as a candidate of a WCCC source (Wu et al. 2019b).

The main concept of SCCC is that the sulfur ions and atoms released from grain surfaces under the effect of shocks will mix with cold gas components and drive a new generation of carbon chain molecules, especially the sulfur-bearing ones such as $\mathrm{C}_{3} \mathrm{~S}$. The criteria of SCCC include relatively weak $\mathrm{N}$-bearing CCM emissions in contrast to strong $\mathrm{C}_{3} \mathrm{~S}$ emissions and signs of developed shock regions. Our model (Wu et al. 2019a) forecasts that, in sources where the SCCC mechanism works most efficiently, the abundance of $\mathrm{C}_{3} \mathrm{~S}\left(\mathrm{X}\left(\mathrm{C}_{3} \mathrm{~S}\right)\right)$ will not only exceed that of $\mathrm{HC}_{7} \mathrm{~N}$ $\left(\mathrm{X}\left(\mathrm{HC}_{7} \mathrm{~N}\right)\right)$, but also that of $\mathrm{HC}_{5} \mathrm{~N}\left(\mathrm{X}\left(\mathrm{HC}_{5} \mathrm{~N}\right)\right)$. The observations presented by $\mathrm{Wu}$ et al. (2019a) show that so far known three SCCC sources (IRAS 20582+7724 (I20582), L1221 and L1251-IRS3) all have $N\left(\mathrm{C}_{3} \mathrm{~S}\right)<N\left(\mathrm{HC}_{5} \mathrm{~N}\right)$, while L1251-IRS3 possesses a $\mathrm{C}_{3} \mathrm{~S}$ column density closest to but still not exceeding that of $\mathrm{HC}_{5} \mathrm{~N}$.

In this work, we further search for and examine SCCC sources in the L1251 region (see Table 1), using the Effelsberg $100 \mathrm{~m}$ telescope in K-band (18-26 GHz). We mapped multiple lines towards the most promising SCCC candidate (L1251-1) located in the tail of the L1251 region, using the Purple Mountain Observatory (PMO) $13.7 \mathrm{~m}$ and the Nobeyama Radio Observatory (NRO) $45 \mathrm{~m}$ tele- scopes in the $3 \mathrm{~mm}$ band as well as the James Clerk Maxwell Telescope (JCMT) in CO $J=3-2$ (see Sect. 2). In Sect. 3, we introduce L1251, including its morphology and structure as well as the star formation activities in L1251-A. The results of our search for new SCCC sources in the L1251 region are presented in Sect. 4, while results of further mapping observations towards L1251-A are presented in Sect. 5. In Sect. 6, we discuss filamentary structures in L1251-A and SCCC related phenomena in this region. Sect. 7 provides a summary.

\section{OBSERVATIONS}

\subsection{Effelsberg}

Single point observations of L1251-1, L1251-2 and L1251-3 were performed during 2018 Jan 29 to 2018 Feb 2 using the Effelsberg $100 \mathrm{~m}$ telescope (see Table 1 and Fig. 1 for their coordinates and locations) in the L1251 region. A Kband (18-26 GHz) HEMT receiver with two polarizations ( $\mathrm{LCP} / \mathrm{RCP})$ was used as the frontend. The lines covered by the K-band are shown in Table 2 . Integration times ranged from 40 to $80 \mathrm{~min}$ utes, depending on the system temperature and the strength of the emission lines. The main beam efficiency ranges from 0.61 to 0.79 depending on the frequency ${ }^{1}$. Flux calibration was accurate to $10 \%$, estimated by observing the standard source NGC7027 (Ott et al. 1994). The pointing accuracy was better than $5^{\prime \prime}$. The half power beam width (HPBW) of the telescope at the observed frequency was approximately $40^{\prime \prime}(0.06 \mathrm{pc}$ assuming a distance of $300 \mathrm{pc})$. Four subbands, WFF4 (18-20.5 $\mathrm{GHz})$, WFF3 (20-22.5 GHz), WFF2(21.6-24.1) and WFF1(23.5-26), can simultaneously cover the whole K-band (18-26 GHz). All spectra were smoothed to a channel spacing of about $0.5 \mathrm{~km} \mathrm{~s}^{-1}$. The 1- $\sigma$ rms noise varied from one to several tens of $\mathrm{mK}$, mainly because of different on-source integration times for our targets.

\footnotetext{
${ }^{1}$ https://eff100mwiki.mpifr-bonn.mpg.de/doku.php?id= information_for_astronomers:rx:s14mm
} 
For unknown reasons, the spectra of WFF3 are shifted redwards by approximately $1 \mathrm{~km} \mathrm{~s}^{-1}$. These misalignments are systematic for all the observed sources and both the two lines c- $\mathrm{C}_{3} \mathrm{H}_{2} 2_{2,0}-2_{1,1}$ and $\mathrm{HC}_{5} \mathrm{~N} J=8-7$ located within WFF3. Therefore, the WFF3 spectra measured were shifted bluewards by two channels.

\section{2. $P M O 13.7 \mathrm{~m}$}

$\mathrm{C}_{2} \mathrm{H} N=1-0, \mathrm{~N}_{2} \mathrm{H}^{+} J=1-0$ and CS $J=2-$ 1 were mapped towards L1251-A (see Fig. 1) in the On-The-Fly (OTF) mode. The maps cover an area of $20^{\prime} \times 10^{\prime}$ and using the PMO $13.7 \mathrm{~m}$ telescope. The PMO $13.7 \mathrm{~m}$ telescope employs a ninebeam Superconducting Spectroscopic Array Receiver (SSAR) as the front end in sideband separation mode (see Shan et al. 2012). A Fast Fourier Transform Spectrometer (FFTS) was employed as the backend, which has a total bandwidth of $1 \mathrm{GHz}$ and 16384 channels, providing a velocity resolution of $\sim 0.2 \mathrm{~km} \mathrm{~s}^{-1}$ (see Table 2). Typical system temperatures are $200 \mathrm{~K}$ and $120 \mathrm{~K}$ for the upper and lower sidebands respectively. The half-power beam width and the main beam efficiency $(\eta)$ are about $56^{\prime \prime}(0.08 \mathrm{pc})$ and $0.5^{2}$, respectively.

\subsection{NRO $45 \mathrm{~m}$}

We applied OTF mapping observations of the $\mathrm{HCO}^{+} J=1-0$, SO $J_{N}=2_{2}-1_{1}, \mathrm{HC}_{3} \mathrm{~N} J=11-$ $10, J=10-9$ and $\mathrm{C}^{18} \mathrm{O} J=1-0$ transitions towards L1251-A (Fig. 1) using the $\mathrm{NRO}^{3} 45 \mathrm{~m}$ telescope during 2019 May 8 th to 14 th. The size of the mapped region is $25^{\prime} \times 10^{\prime}$. A four-beam receiver (FOREST; Minamidani et al. 2016) was adopted as frontend and SAM 45 (Kamazaki et al. 2012) was adopted as backend. $\mathrm{HCO}^{+} J=1-0$, SO $J_{N}=2_{2}-1_{1}, \mathrm{HC}_{3} \mathrm{~N} J=11-10$ and $J=10-$ 9 were observed simultaneously. The half-power beam width (HPBW) and beam efficiency of FOR-

${ }^{2}$ http://english.dlh.pmo.cas.cn/fs

${ }^{3}$ The Nobeyama Radio Observatory is a branch of the National Astronomical Observatory of Japan, National Institutes of Natural Sciences.
EST at $86 \mathrm{GHz}$ were about $19^{\prime \prime}(0.028 \mathrm{pc})$ and 0.5 , respectively. Spectra obtained with orthogonal linear polarizations were averaged to improve the signal-to-noise ratio (SNR). The system temperature varied from $150 \mathrm{~K}$ to $300 \mathrm{~K}$ depending on the zenith angles and weather conditions during the observations. The data were baseline extracted and gridded using the package NOSTAR ${ }^{4}$ provided by the NRO. The spectra were smoothed to a velocity resolution of $\sim 0.2 \mathrm{~km} \mathrm{~s}^{-1}$ and gridded in $10^{\prime \prime}$ intervals. The resulting noise levels in the main beam brightness temperature $\left(T_{\mathrm{mb}}\right)$ scale varied from 0.05 to $0.17 \mathrm{~K}$ for different lines, depending on integration times and system temperatures.

\subsection{JCMT HARP}

We mapped L1251-A (Fig. 1) employing the JCMT OTF mode in CO $J=3-2$, under the project ID M19BP0575. The HARP receiver was adopted. The observations were performed in November 14, 2019 , with an on-source time of $\sim 30$ minutes. The size of the mapped region is $20^{\prime} \times 10^{\prime}$. The half power beam width is approximately $14^{\prime \prime}$. We smoothed and gridded the data cube with a pixel size $15^{\prime \prime} \times 15^{\prime \prime}$ and a channel spacing of $0.2 \mathrm{~km} \mathrm{~s}^{-1}$, to improve the signal-to-noise ratio. The 1 sigma rms noise level is $0.5 \mathrm{~K}$.

\section{OUR TARGETED SOURCES IN THE L1251 REGION}

The dark cloud L1251 (Lynds 1962) is located in the molecular ring of the Cepheus Flare and was found to be a site of recent low mass star formation (Sato \& Fukui 1989; Sato et al. 1994; Rosvick \& Davidge 1995; Kim et al. 2015).

The distance is adopted as $300 \pm 30 \mathrm{pc}$ derived from the 3-dimensional (3D) dust map based on Gaia (Green et al. 2019). It is consistent with the values of $300 \pm 50$ pc adopted by Kun \& Prusti

\footnotetext{
${ }^{4}$ https://www.nro.nao.ac.jp/ nro45mrt/html/obs/otf/exporte. html

${ }^{5}$ http://www.cadc-ccda.hia-iha.nrc-cnrc.gc.ca/en/search/ ?Observation.proposal.id=M19BP057\&Observation. collection $=\mathrm{JCMT}$
} 
(1993) and 330 \pm 30 pc adopted by Balázs et al. (2004) and Wu et al. (2019a). Based on CO observations, the L1251 cloud was divided into five substructures by Sato et al. (1994), and these substructures were designated by letters from A to $\mathrm{E}$ (see the top panel of Fig. 1). The L1251 cloud is elongated in east-west direction, consisting of a dense "head" in the east and a diffuse "tail" in the west (Sato \& Fukui 1989; Sato et al. 1994).

Four low-luminosity young stellar objects (Dunham et al. 2008) are located in the tail L1251-A, named as IRS1-4 from west to east. These were identified by the $c 2 d$ team based on the colors in Spitzer bands (Dunham et al. 2008; Evans et al. 2009; Enoch et al. 2009). The two sources, denoted as L1251-1 and L1251-2 in this work, contain IRS1 and IRS4, respectively (see Fig. 1). The SCCC source denoted as L1251A (referred as L1251-IRS3 in this work) in Wu et al. (2019a) contains IRS3. A well-collimated jet in north-south direction associated with IRS3 (see also Sect. 5.1) can be explained with a small precession angle and a long stellar pulsating period (Lee et al. 2010). L1251-3 is located in the head of L1251 (Fig. 1), and it is also associated with two young stellar objects (YSOs) identified by Spitzer data. Our targets L1251-1, L1251-2, and L1251-3 are all candidates of very low luminosity objects (VeLLOs) with $L_{i n t}<0.1 L_{\odot}$, but may not be deeply embedded sources (Dunham et al. 2008).

\subsection{YSO classfication and their continuum counterparts}

Continuum fluxes in Spitzer bands $(3.6 \mu \mathrm{m}, 4.5$ $\mu \mathrm{m}, 5.8 \mu \mathrm{m}, 8.0 \mu \mathrm{m}, 24 \mu \mathrm{m}$ and $70 \mu \mathrm{m}$ ), as well as other near infrared to milimetre continuum data with wavelengths ranging from $1.25 \mu \mathrm{m}$ to $850 \mu \mathrm{m}$ were taken from the literature, and are listed in Table 1 .

A near-infrared 2MASS point source counterpart is found near IRS2 within 3" (900 AU). All four YSOs associated with L1251-A have counterparts within 5" (1500 AU) in Herschel's PACS Point Source Catalogue. The $70 \mu \mathrm{m}$ fluxes of the four
YSOs obtained by Herschel are about 1.2 times of the values obtained by Spitzer. This difference may be introduced by different source extracting algorithms. IRS-3 and IRS-4 have been mapped by $\mathrm{Wu}$ et al. (2007) at $350 \mu \mathrm{m}$ using SHARC-II mounted on the Caltech Submillimeter Observatory (CSO). Three SCUBA-2 cores identified by the JCMT Gould Belt Survey (Pattle et al. 2017) (with core number 91, 82 and 57) are centered close to (< $\left.20^{\prime \prime}\right)$ IRS2, IRS3 and IRS4, respectively. These angular separations are comparable with the full width at half maximum (FWHM) source sizes $\left(\sim 30^{\prime \prime}\right)$ and SCUBA beam sizes $\left(\sim 10^{\prime \prime}\right.$ at $450 \mu \mathrm{m}$ and $\sim 15^{\prime \prime}$ at $\left.850 \mu \mathrm{m}\right)$. The SCUBA-2 core 95 is separated from L1251-3 by $\sim 1^{\prime}$. The SCUBA-2 core 71 (Pattle et al. 2017) locates between IRS1 and IRS2, with IRS1 in its southwestern margin and IRS2 in the eastern margin. All four YSOs in L1251-A except IRS1 have continuum detection at $1.2 \mathrm{~mm}$ (Kauffmann et al. 2008) by the Max-Planck Millimetre Bolometer (MAMBO) at the IRAM 30 m telescope.

These YSOs are classified based on color criteria and spectral energy distribution (SED) fittings (Appendix A). IRS3 and IRS4 are classified as Class 0/I and Class II respectively. IRS1 and IRS2 are classified as Class Flat. L1251-3 contains a Class II young stellar object.

\subsection{Large scale structures}

The map of the L1251 region from Herschel Space Telescope data consists of hierarchical filaments and filamentary sub-structures (see Fig. 1). Not only the tail of L1251, but also the "head" of L1251 can be visually divided into several filamentary sub-structures. To more accurately unveil the filamentary structures in this region, we obtained a surface density as well as a temperature map of the dust in the L1251 region from the Herschel images. The fitting procedures are described in Appendix B (see Fig. 1).

\subsubsection{Extracting filaments}


The filamentary structure is extracted from the surface density map using FilFinder ${ }^{6}$ (a python package developed by Koch \& Rosolowsky (2015)) to identify and analyse filaments. The skeleton of the extracted filamentary structures is shown in the lower panel of Fig. 1. Six filamentary sub-structures, named as F1 to F6 were identified in L1251 and L1247. L1251-A is associated with F1. The U-shaped "head" (Sato et al. 1994) is made up by three distinguished sub-filaments, F2, F3 and F4. The southwestern ends of F2 and F3, together with the eastern end of $\mathrm{F} 1$, converge to a hub region that we called $\mathrm{H}$ (see the lower panel of Fig. 1). The hub region is much less dense than the main parts of the three sub-filaments.

The multiforked fibrous sub-structure F6 at the western side of L1251-A (see Fig. 1), does not belong to L1251 but is a part of L1247. However, a slender and curved sub-filament F5 with very low surface density (about one tenth of the values for the other sub-filaments) is found connecting F1 and F6. L1251-A (F1) is regarded as the western tail of the L1251 region by Sato et al. (1994) based on CO images. The F1-6 make up a dragon-like skeleton, in which F1 is the spine instead of the tail of the whole structure consisting of L1251 and L1247.

\subsubsection{Substructures in L1251-A}

In the L1251-A region, there is a side branch with a shape analogous to a letter " $\mathrm{S}$ " rotated counterclockwise by almost 90 degrees (Figs. 1 and 2). We named this side branch intertwining with the main branch as F1-S. The segment of the main branch tangled by F1-S is denoted as F1-M, which contains the 4 YSOs in L1251-A. F1-M and F1$\mathrm{S}$ make up a lying down " 8 " with two cavities. The eastern parts of F1-M and F1-S surrounding the eastern cavity $(\mathrm{C} 1)$ are denoted as F1-ME and F1-SE, while the western parts surrounding the

\footnotetext{
${ }^{6}$ https://github.com/e-koch/FilFinder
}

western cavity (C2) are denoted as F1-MW/F1-SW (See Fig. 1 and Fig. 2 for the details).

\section{PARAMETERS OF THE RADIO K-BAND LINES}

The spectra obtained in K-band using the Effelsberg $100 \mathrm{~m}$ telescope include $\mathrm{HC}_{3} \mathrm{~N} J=2-1$, $\mathrm{HC}_{5} \mathrm{~N} J=9-8,8-7$ and $7-6, \mathrm{C}_{3} \mathrm{~S} J=4-3, \mathrm{NH}_{3}$ $(1,1), \mathrm{NH}_{3}(2,2), \mathrm{C}_{4} \mathrm{H} N=2-1 J=5 / 2-3 / 2 F=$ $3-2, \mathrm{c}-\mathrm{C}_{2} \mathrm{H}_{2} 1_{1,0}-1_{0,1}$ and $\mathrm{c}-\mathrm{C}_{2} \mathrm{H}_{2} 2_{2,0}-2_{1,1}$. These spectra are shown in Fig. 3.

The lines observed in K-band are fitted with single Gaussian profiles, except for $\mathrm{NH}_{3}(1,1)$, which has been fitted with the hyperfine structures (HFS; see Sect. 4.1). We also tried to apply HFS fitting to $\mathrm{HC}_{3} \mathrm{~N} J=2-1$, but excitation temperatures are not available because $\mathrm{HC}_{3} \mathrm{~N} J=2-1$ is optically thin in most sources (Sect. 4.1; see also Levshakov et al. (2013)). The hyperfine structure of $\mathrm{HC}_{3} \mathrm{~N}$ $J=2-1$, except for the blended $F=3-2$ and $F=$ 2-1 features, is only marginally detected in L12512, and not detected in L1251-1 and L1251-3. The peak optical depth of the unsplit $\mathrm{HC}_{3} \mathrm{~N} J=2-1$ (assuming the central frequencies of all hyperfine structures are aligned) of L1251-2 is $0.20 \pm 0.15$, given by the standard CLASS $\mathrm{HFS}^{7}$ procedure. We conclude that the $\mathrm{HC}_{3} \mathrm{~N} J=2-1$ lines are optically thin for the observed sources. The fitted parameters are listed in Table 3.

The column densities of $\mathrm{H}_{2}\left(N^{\text {dust }}\left(\mathrm{H}_{2}\right)\right)$ and dust temperatures ( $T_{\text {dust }}$ ) of L1251-1, L1251-2 and L1251-3 are extracted from the surface density and dust temperature maps (see Sect. 3.2), and are smoothed to the $40^{\prime \prime}$ beam size of the Effelsberg telescope. The derived $N^{\text {dust }}\left(\mathrm{H}_{2}\right)$ and $T_{\text {dust }}$ are listed in Table 5.

\section{1. $\mathrm{NH}_{3}$ and linear $\mathrm{CCMS}$}

Optical depths $(\tau)$, excitation temperatures $\left(T_{\mathrm{ex}}\right)$ of $\mathrm{NH}_{3}(1,1)$, rotational temperatures $\left(T_{\text {rot }}\right)$ and column densities of $\mathrm{NH}_{3}$, as well as the volume density of $\mathrm{H}_{2}$ derived from $\mathrm{NH}_{3}$ emissions

\footnotetext{
${ }^{7}$ https://www.iram.fr/IRAMFR/GILDAS/
} 
$\left(n^{N H_{3}}\left(\mathrm{H}_{2}\right)\right)$ have been calculated (see Appendix C). The fitted line parameters of $\mathrm{NH}_{3}$ are listed in Table 4. The derived parameters for $\mathrm{NH}_{3}, T_{\mathrm{ex}}\left(\mathrm{NH}_{3}\right)$, $T_{\text {rot }}\left(\mathrm{NH}_{3}\right)$ and $n^{N_{3}}\left(\mathrm{H}_{2}\right)$ are listed in Table 5.

All the species $\mathrm{HC}_{3} \mathrm{~N}, \mathrm{HC}_{5} \mathrm{~N}, \mathrm{C}_{3} \mathrm{~S}$ and $\mathrm{C}_{4} \mathrm{H}$ are linear carbon-chain molecules. To calculate the column densities, excitation temperatures should be known. Unfortunately, the three rotational lines of $\mathrm{HC}_{5} \mathrm{~N}$ can not be used to derive the excitation temperatures $T_{\mathrm{ex}}$, since they all have upper level energies lower than $6 \mathrm{~K}$. The $\mathrm{HC}_{5} \mathrm{~N} J=9-8$ line has been adopted to calculate the column density of $\mathrm{HC}_{5} \mathrm{~N}$, since it has the highest SNR. The excitation temperatures are assumed to be identical with the rotational temperatures of $\mathrm{NH}_{3}$, which are close to the dust temperatures, with differences smaller than $1 \mathrm{~K}$. The column densities of these carbonchain species are calculated under the assumption of local thermal equilibrium (LTE), using Eq. C6 in Appendix C, with the line strength $S$ and partition function $Q_{\text {rot }}$ quoted from Splatalogue ${ }^{8}$. If the excitation temperature is changed by $2 \mathrm{~K}$, the calculated column density will change by less than 20 percent. The dipole moment of $\mathrm{C} 4 \mathrm{H}$ is adopted as $0.9 \mathrm{D}$. If the value of 2.10 D is adopted (Oyama et al. 2020) the evalued column densities will be a factor of 6 smaller than the presented ones.

The column densities and abundances of $\mathrm{NH}_{3}$ and CCMs are listed in Table 6.

\section{2. $c-\mathrm{C}_{3} \mathrm{H}_{2}$}

Cyclopropenylidene $\left(\mathrm{c}-\mathrm{C}_{3} \mathrm{H}_{2}\right)$, the first detected interstellar organic ring molecule, is a typical constituent of the dense interstellar medium (Thaddeus et al. 1985; Matthews \& Irvine 1985; Vrtilek et al. 1987). $\quad$ c- $\mathrm{C}_{3} \mathrm{H}_{2}$ has $C_{2 v}$ symmetry. Two $\mathrm{H}$ atoms are coupled to generate the ortho (nuclear spin $=1$ ) and para (nuclear spin $=0$ ) species of c- $\mathrm{C}_{3} \mathrm{H}_{2}$ with spin statistical weights of 3 and 1 , respectively (Park et al. 2006). Because of the Pauli exclusion principle, para $\mathrm{c}-\mathrm{C}_{3} \mathrm{H}_{2}$ is charac-

\footnotetext{
${ }^{8}$ http://www.cv.nrao.edu/php/splat/
}

terized by even $K_{a}+K_{c}$, and ortho $\mathrm{c}-\mathrm{C}_{3} \mathrm{H}_{2}$ by odd $K_{a}+K_{c}$ values. The abundance ratios between the ortho $\mathrm{c}-\mathrm{C}_{3} \mathrm{H}_{2}\left(\mathrm{o}-\mathrm{C}_{3} \mathrm{H}_{2}\right)$ and para $\mathrm{c}-\mathrm{C}_{3} \mathrm{H}_{2}\left(\mathrm{p}-\mathrm{C}_{3} \mathrm{H}_{2}\right)$ in TMC-1 are all larger than one, and can reach 3 for evolved cores. The ortho-to-para ratio (o/p ratio) of $\mathrm{c}-\mathrm{C}_{3} \mathrm{H}_{2}$ is $3.1 \pm 0.4$ in L1257 (Takakuwa et al. 2001; Yoshida et al. 2015). Park et al. (2006) modeled the $\mathrm{o} / \mathrm{p}$ ratios of $\mathrm{c}-\mathrm{C}_{3} \mathrm{H}_{2}$, and found that the $\mathrm{o} / \mathrm{p}$ ratios of $\mathrm{c}-\mathrm{C}_{3} \mathrm{H}_{2}$ can reach 3 if exchange processes involving $\mathrm{H}^{+}$and protonating ions $\mathrm{HX}^{+}$ were considered, even if $\mathrm{H}_{2}$ (the precursor of $\mathrm{c}$ $\mathrm{C}_{3} \mathrm{H}_{2}$ ) is overwhelmingly para.

Fig. 14 in Appendix D shows the energy diagram of $\mathrm{c}-\mathrm{C}_{3} \mathrm{H}_{2}$. The states $2_{2,0}$ and $2_{1,1}$ of para c- $\mathrm{C}_{3} \mathrm{H}_{2}$ have similar upper-level energies ( $\left.\sim 9 \mathrm{~K}\right)$. The transition between $2_{1,1}$ and $1_{1,1}$ is forbidden ${ }^{9}$, and c- $\mathrm{C}_{3} \mathrm{H}_{2}$ in state $2_{1,1}$ can only transit to $2_{0,2}$, with a spontaneous emission coefficient $2.679 \times 10^{-6} \mathrm{~s}^{-1}$. However, the spontaneous emission coefficient between $2_{2,0}$ and $1_{1,1}$ is large $\left(5.354 \times 10^{-5} \mathrm{~s}^{-1}\right)$. Thus the state $2_{2,0}$ is more difficult to populate than $2_{1,1}$, and the population ratio between $2_{2,0}$ and $2_{1,1}$ is small with excitation temperatures lower than the temperature of the background radiation. $\quad c-\mathrm{C}_{3} \mathrm{H}_{2}$ in state $2_{1,1}$ will be pumped to $2_{2,0}$ through absorption of background photons (e.g. the cosmic microwave background), and further transit to $1_{1,1}$. This is the reason why c- $\mathrm{C}_{3} \mathrm{H}_{2} 2_{2,0}-2_{1,1}$ shows absorption features in all of our detected sources. With a typical $\mathrm{H}_{2}$ volume density $n=10^{5} \mathrm{~cm}^{-3}$, kinetic temperature $T_{\text {kin }}=10 \mathrm{~K}$ and $\mathrm{c}-\mathrm{C}_{3} \mathrm{H}_{2}$ column density $N=10^{13} \mathrm{~cm}^{-2}$, the excitation temperatures of $\mathrm{c}-\mathrm{C}_{3} \mathrm{H}_{2} 2_{2,0}-2_{1,1}$ and $1_{1,0}-1_{0,1}$ from RADEX are $1.2 \mathrm{~K}$ and $5.4 \mathrm{~K}$, respectively.

We assume a constant $\mathrm{o} / \mathrm{p}$ ratio of 3 here to fit the $\mathrm{c}-\mathrm{C}_{3} \mathrm{H}_{2} 1_{1,0}-1_{0,1}$ (ortho type) emission and the c- $\mathrm{C}_{3} \mathrm{H}_{2} 2_{2,0}-2_{1,1}$ (para type) absorption lines, following the fitting method described in Appendix $D$. The $\mathcal{R}$ values (the ratio between the intensity of para c- $\mathrm{C}_{3} \mathrm{H}_{2} 2_{2,0}-2_{1,1}$ and that of ortho $\mathrm{c}-\mathrm{C}_{3} \mathrm{H}_{2}$ $1_{1,0}-1_{0,1}$ ) for L1251-1, L1251-2 and L1251-3 are

\footnotetext{
${ }^{9}$ https://home.strw.leidenuniv.nl/ moldata/
} 
$-0.38,-0.22$ and -0.40 , respectively. The volume densities $\left(n^{c-C_{3} H_{2}}\left(\mathrm{H}_{2}\right)\right)$ of L1251-1 and L1251-3 can not be well constrained through Eq. D13 in Appendix D and the values are adopted as $10^{4}$ $\mathrm{cm}^{-3}$, the value below which the $\mathcal{R}$ value will be little affected by $n$. The volume density of L1251-2 is estimated as $3 \times 10^{5} \mathrm{~cm}^{-3}$ from $\mathcal{R}$ using Eq. D13. However, we still allow volume density to vary and fit the column density of $\mathrm{c}-\mathrm{C}_{3} \mathrm{H}_{2}$ and volume density simultaneously. The kinetic temperatures are adopted as $T_{\text {rot }}\left(\mathrm{NH}_{3}\right)$ for the fittings (Appendix D). The column densities of $\mathrm{c}_{-} \mathrm{C}_{3} \mathrm{H}_{2}$ for L12511, L1251-2 and L1251-3 given by the fittings are $6.7 \times 10^{13}, 1.7 \times 10^{13}$ and $4.8 \times 10^{13} \mathrm{~cm}^{-2}$, respectively. The fitted $n^{c-C_{3} H_{2}}\left(\mathrm{H}_{2}\right)$ is $2.2 \times 10^{5} \mathrm{~cm}^{-3}$ for L1251-2. It should be noted that the emission model described in Appendix D assumes that the emissions are from a uniform medium. The column densities of $\mathrm{c}^{-} \mathrm{C}_{3} \mathrm{H}_{2}$ for L1251-1 and L1251-3 will be over-estimated because of the under estimations of the volume densities. The c- $\mathrm{C}_{3} \mathrm{H}_{2} 1_{1,0^{-}}$ $1_{0,1}$ emission may mainly originate from the inner denser regions, while the absorption features of c$\mathrm{C}_{3} \mathrm{H}_{2} 2_{2,0}-2_{1,1}$ may originate from the outer diffuse regions, where these molecules may also be quite abundant (Madden et al. 1989) but can not be effectively populated.

\subsection{Signposts of SCCC}

L1251-1 and L1251-3 both have integrated intensities of $\mathrm{C}_{3} \mathrm{~S} J=4-3$ larger than those of $\mathrm{HC}_{5} \mathrm{~N} J$ = 9-8. In contrast, L1251-2 shows much weaker emission of $\mathrm{C}_{3} \mathrm{~S} J=4-3$ compared with $\mathrm{HC}_{5} \mathrm{~N}$ $J=9-8$. Since $\mathrm{C}_{3} \mathrm{~S}$ and $\mathrm{HC}_{5} \mathrm{~N}$ are both linear molecules with similar electric dipole moments (3.6 and 4.33 respectively), their abundance ratio can be approximately represented by the integrated intensity ratio between their lines at similar frequency. This criterion can help us quickly find candidate sources with $\mathrm{N}\left(\mathrm{C}_{3} \mathrm{~S}\right)>\mathrm{N}\left(\mathrm{HC}_{5} \mathrm{~N}\right)$. The abundance ratios between $\mathrm{C}_{3} \mathrm{~S}$ and $\mathrm{HC}_{5} \mathrm{~N}$ are $2.1 \pm 0.4$ and $1.2 \pm 0.2$ for L1251-1 and L1251-3, but $0.4 \pm 0.1$ for L1251-2. Such high abundances of $\mathrm{C}_{3} \mathrm{~S}$ in L1251-1 and L1251-3 are quite rare in cold clouds and have not been measured in star-forming regions before (see Sect. 6.3).

For L1251-2 and L1251-3, the column density ratios between $\mathrm{HC}_{3} \mathrm{~N}$ and $\mathrm{HC}_{5} \mathrm{~N}$ $\left(N\left(\mathrm{HC}_{3} \mathrm{~N}\right) / N\left(\mathrm{HC}_{5} \mathrm{~N}\right)\right)$ are about 4 , similar to the value of starless cores and common outflow sources (Takano et al. 1998; Benedettini et al. 2012; Taniguchi et al. 2016; Law et al. 2018). L1251-1 has a larger $N\left(\mathrm{HC}_{3} \mathrm{~N}\right) / N\left(\mathrm{HC}_{5} \mathrm{~N}\right)$ ratio of $8 \pm 1$ (see Fig. 4), which is closer to the values of other SCCC sources (Wu et al. 2019a).

Abundant $\mathrm{C}_{4} \mathrm{H}$, especially derived from its high excitation lines with $\Delta E_{u}>20 \mathrm{~K}$, is the key to study the heating of the ambient medium by YSOs and to investigate the warm carbon chain chemistry , as suggested by Sakai et al. (2008b, 2009). From our observations, L1251-1 and L1251-3 have larger abundance ratios between $\mathrm{C}_{4} \mathrm{H}$ and $\mathrm{HC}_{3} \mathrm{~N}$ (9.0 and 8.6) than that of L1251-2 (4.5). The sources detected in L1251 have similar column densities of $\mathrm{C}_{4} \mathrm{H}$ compared to the WCCC source L1527 $\left(8.8 \pm 0.5 \times 10^{13} \mathrm{~cm}^{-3}\right)$. The upper level energy of the detected $\mathrm{C}_{4} \mathrm{H}$ line is low (see Table 2), and a contribution of the emission from an extended component can not be entirely ruled out. Nevertheless, the WCCC theory is a possible explanation for the large hydrocarbon abundances, as suggested by Cordiner et al. (2011) based on their observations near L1251-IRS3. However, their measured position is one arcmin farther away from the stellar source than the one observed by $\mathrm{Wu}$ et al. (2019a), which shows that the precursors of the shocked region in L1251-IRS3 may be WCCC regions. The abnormally high abundances of $\mathrm{C}_{3} \mathrm{~S}$ we detected imply an influence of shocks in the parent gas component with high abundances of hydrocarbons.

For L1251-1 (IRS-1), the line width of $\mathrm{C}_{3} \mathrm{~S} J=4$ $3\left(2.5 \mathrm{~km} \mathrm{~s}^{-1}\right)$ is obviously higher than the values $\left(<1.4 \mathrm{~km} \mathrm{~s}^{-1}\right)$ of transitions of other species except $\mathrm{C}_{4} \mathrm{H}$ (see Table 4), even considering the low velocity resolution $\left(\sim 0.5 \mathrm{~km} \mathrm{~s}^{-1}\right)$ of the observed spectra. This large line width is introduced by the 
red velocity component in the $\mathrm{C}_{3} \mathrm{~S}$ spectrum (see Fig. 3), which we suggest as an evidence of outflow activity driven by IRS-1 (see also Sect. 5.1). The relatively large line width of $\mathrm{C}_{4} \mathrm{H}\left(1.8 \mathrm{~km} \mathrm{~s}^{-1}\right)$ may also be affected by star formation activities.

Overall, L1251-1 characterized by $N\left(\mathrm{C}_{3} \mathrm{~S}\right)>N\left(\mathrm{HC}_{5} \mathrm{~N}\right)$ is a clear SCCC source that were looking for. L1251-3 has $N\left(\mathrm{C}_{3} \mathrm{~S}\right)$ slightly larger than $N\left(\mathrm{HC}_{5} \mathrm{~N}\right)$ but lacks of mapping observagtions and is identifed as a candidate SCCC source.

\section{MAPPING ANALYSIS TOWARDS L1251-A}

\subsection{Outflows traced by $C O \mathrm{~J}=3-2$ lines}

Features of outflows are pronounced in the L1251-A region traced by $\mathrm{CO} J=3-2$ as shown in Fig. 5.

\subsubsection{Outflows from IRS3}

A bipolar $\mathrm{CO} J=3-2$ jet originating from IRS3 extending in north-south direction has been detected. The blue part of the jet consists of two bullets. The bullet close to IRS3 corresponds to the slender blue finger in the position-velocity $(\mathrm{P}-\mathrm{V})$ map (near the zero offset of the lower right panel of Fig. 5), and the other one located more than $2^{\prime}(0.17 \mathrm{pc})$ away from IRS 3 corresponds to a blueward convex bulge (see the lower right panel of Fig. 5). The jet traced by $\mathrm{CO} J=3-2$ is similar to that previously revealed by a CO $J=2-1$ image (Lee et al. 2010). A jet with a small precession angle and long pulsating period was introduced by Lee et al. (2010) to explain their observations.

Among the four YSOs in L1251-A, only IRS3 is accompanied by characteristics of strong bipolar jets in Spitzer maps. From the $\mathrm{CO} J=3-2$ observation, the red and blue lobes are spatially separated by $6^{\prime}$ with a velocity separation of about $8 \mathrm{~km} \mathrm{~s}^{-1}$. The derived dynamical timescale is about $6 \times 10^{4} \mathrm{yr}$, which is consistent with the value $5 \times 10^{4}$ yr given by Lee et al. (2010). This outflow activity was thought to be the driving source of SCCC around IRS3 (Wu et al. 2019a). The high abundance of $\mathrm{C}_{3} \mathrm{~S}$ relative to $\mathrm{HC}_{5} \mathrm{~N}$ in L12511 (IRS1), may originate from outflows driven by IRS1. The outflow activity has extinguished now, since in our model (Wu et al. 2019a), the enhancement of $\mathrm{C}_{3} \mathrm{~S}$ will not lead to $\mathrm{X}\left[\mathrm{C}_{3} \mathrm{~S}\right]>\mathrm{X}\left[\mathrm{HC}_{5} \mathrm{~N}\right]$ till $10^{5} \mathrm{yr}$ after the start of the enrichment of the gas phase by sulfur from shocks induced by outflows. The SCCC sources found in Wu et al. (2019a) all have outflows with timescales smaller than $10^{5}$ $\mathrm{yr}$, and are thus in an earlier stage of SCCC with $X\left[\mathrm{C}_{3} \mathrm{~S}\right]>X\left[\mathrm{HC}_{7} \mathrm{~N}\right]$ but $X\left[\mathrm{C}_{3} \mathrm{~S}\right]<X\left[\mathrm{HC}_{5} \mathrm{~N}\right]$.

\subsubsection{Outflows from IRS4}

A blue lobe in south-east direction is detected in CO $J=3-2$ around IRS4 (L1251-2; see Fig. 5). It is more compact than that detected in $\mathrm{CO} J=2-$ 1 using the six-meter telescope of the Seoul Radio Astronomy Observatory (SRAO) (Kim et al. 2019). However, a redward instead of the blueward lobe was also detected towards IRS4 using the SRAO (Lee et al. 2010). In our P-V map (upper right panel of Fig. 5), a blue finger at an offset of $+1^{\prime}$ is associated with IRS4. A similar but red finger at the same offset indicates a small angle between the direction of the jet and the line of sight. This may explain why the blue and red lobe are coincident. It should be noted that a small red component with a velocity range from $-1 \mathrm{~km} \mathrm{~s}^{-1}$ to $0 \mathrm{~km} \mathrm{~s}^{-1}$ can be seen from the spectra of CO $J=$ 2-1 (Kim et al. 2019), and it may contribute in a non-ignorable way to the intensity of the red lobe integrated from $-1 \mathrm{~km} \mathrm{~s}^{-1}$ to $5 \mathrm{~km} \mathrm{~s}^{-1}$ by Lee et al. (2010).

The clump located in the northwest of IRS4, denoted as IRS4-MNW in the left panel of Fig. 5, is accompanied by both blue and red lobes. These two lobes correspond to the blue and red fingers in the P-V map (upper right panel of Fig. 5) with an offset of approximate $1^{\prime}$. The three-color image comprised of IRAC 3.6, 4.5, and $8.0 \mu \mathrm{m}$ images is shown in Fig. 6. IRS4-MNW is associated with a star-like object at the northwestern edge (22:30:54 +75:14:54), with an infrared spectral index $\alpha_{I R}$ (defined in Appendix A) of 1.24 
(Evans et al. 2009). This object was not classified as a young stellar object because it has a weak Spitzer/MIPS $24 \mu \mathrm{m}$ flux (0.212 mJy) and was not detected in the Spitzer/MIPS $70 \mu \mathrm{m}$ band. Its color and morphology shown in Fig. 6 are similar to the shocked regions driven by IRS3. This object may represent shocked gas.

\subsubsection{Hint for outflows from L1251-1 (IRS1)}

Blue and red wing emissions were detected towards IRS1 in ${ }^{12} \mathrm{CO}$, although at a much weaker level than that in IRS3 (Schwarz et al. 2012). The signature of the red $\mathrm{C}_{3} \mathrm{~S} J=4-3$ wing of L1251-1 shown in Fig. 3 is also consistent with the redder velocity of IRS 1 compared to IRS2 and IRS4 shown in the P-V diagram (Fig. 12). No wing signature of $\mathrm{C}_{3} \mathrm{~S} J=4-3$ is detected in L1251-2 and L1251-3. The red $\mathrm{C}_{3} \mathrm{~S} J=4-3$ wing in IRS1 extends from $-3 \mathrm{~km} \mathrm{~s}^{-1}$ to $0 \mathrm{~km} \mathrm{~s}^{-1}$ and is consistent with the idea that $\mathrm{C}_{3} \mathrm{~S}$ in this region is shock related.

Bipolar jets should be common in this region, and there might also exist episodic jets originating from IRS1 and IRS2, but these putative jets, if they existed at all, are extinguished now.

\subsection{Maps of the PMO $13.7 \mathrm{~m}$}

The integrated intensity maps of CS $J=2-1$, $\mathrm{C}_{2} \mathrm{H} N=2-1 \quad J=3 / 2-1 / 2 F=2-1$ and $\mathrm{N}_{2} \mathrm{H}^{+} J=$ $1-0 F_{1}=2-1$ are shown in Fig. 7 with maximum values of 0.53 (0.03), $0.29(0.02)$ and $0.88(0.04)$ $\mathrm{K} \mathrm{km} \mathrm{s}^{-1}$ around IRS4, respectively. The emission regions of $\mathrm{C}_{2} \mathrm{H}$ and $\mathrm{N}_{2} \mathrm{H}^{+}$are more compact than those of CS, with emission centers shifted northwards. Their shapes are convex and well correlated with the main F1 branch, which runs across the four YSOs. It is consistent with the fact that the northern part of L1251-A is denser (see Fig. 2) and may be more evolved, since $\mathrm{N}_{2} \mathrm{H}^{+}$tends to be enhanced in evolved regions (Caselli et al. 2002; Liu et al. 2019b), while CS $J=2-1$ is usually characterized by more extended emission, which is getting closer to the size of the $\mathrm{CO}$ emission region (Wu 1993). Besides, the abundance of CS tends to be enhanced in turbulent regions, and CS emission is consistent with the chemical effects expected in shocked regions (Hartquist et al. 1980; Zhou et al. 1989; Suzuki et al. 1992; Luo et al. 2019). The depletion of the CS molecule (Vastel et al. 2018; Kim et al. 2020) in the dense region of F1-M will also make the CS emission look more extended.

Assuming the emission lines are optically thin and the excitation temperatures are the same as the dust temperatures of the dense regions, $\sim 10 \mathrm{~K}$ (see Fig. 1 and Table 5), the conversion factors between the line intensities and column densities are $4.7 \times 10^{12}, 5.1 \times 10^{13}$ and $2.4 \times 10^{12} \mathrm{~cm}^{-2}$ per $\mathrm{K} \mathrm{km}$ $\mathrm{s}^{-1}$ for CS $J=2-1, \mathrm{C}_{2} \mathrm{H} J=3 / 2-1 / 2 F=2-1$ and $\mathrm{N}_{2} \mathrm{H}^{+} J=1-0 F_{1}=2-1$, respectively. The ratios between the column densities of $\mathrm{C}_{2} \mathrm{H}$ and $\mathrm{N}_{2} \mathrm{H}^{+}$ $\left(\mathrm{X}\left[\mathrm{C}_{2} \mathrm{H}\right] / \mathrm{X}\left[\mathrm{N}_{2} \mathrm{H}^{+}\right]\right)$have a mean value of 8 with a standard deviation of 3 . The abundance ratios of $\mathrm{N}\left[\mathrm{C}_{2} \mathrm{H}\right] / \mathrm{N}\left[\mathrm{N}_{2} \mathrm{H}^{+}\right]$in the L1251-A region are comparable with the values of typical dark clouds and star-forming regions, but tend to be lower than the typical value ( $>10$ ) of Planck Galactic cold clumps (PGCCs) (Liu et al. 2019b). In starless cores the abundance ratio between $\mathrm{C}_{2} \mathrm{H}$ and $\mathrm{N}_{2} \mathrm{H}^{+}$will decrease as the cores evolve, but it is not well constrained in star forming regions. However, the low values of $\mathrm{N}\left(\mathrm{C}_{2} \mathrm{H}\right) / \mathrm{N}\left(\mathrm{N}_{2} \mathrm{H}^{+}\right)$in L1251-A imply that this region is to overall not in chemically young phase.

\subsection{Maps of the NRO $45 m$}

\subsection{1. $S O$ and $C^{18} O$}

The two cavities ( $\mathrm{C} 1$ and $\mathrm{C} 2$ ) enclosed by the two twisted sub-filaments can also be identified clearly in the emission map of SO $J_{N}=2_{2}-1_{1}$ (see Fig. 8), observed using the NRO $45 \mathrm{~m}$. The dust emission from F1-ME is much stronger than that from F1-SE, while F1-SE shows stronger $\mathrm{SO} J_{N}=$ $2_{2}-1_{1}$ emission. The $\mathrm{C}^{18} \mathrm{O}$ emission region shows a similar morphology to SO $J_{N}=2_{2}-1_{1}$, except that the differences of the emission intensities of $\mathrm{C}^{18} \mathrm{O}$ between F1-SE and F1-ME are more notable than in SO. There are no $\mathrm{C}^{18} \mathrm{O}$ clumps around IRS3 and 
IRS4. F1-ME, with $V_{\mathrm{LSR}}$ of about $-3.8 \mathrm{~km} \mathrm{~s}^{-1}$, is slightly redder than F1-SE, with $V_{\mathrm{LSR}}$ about -4.2 $\mathrm{km} \mathrm{s}^{-1}$. The emission of $\mathrm{SO}$ and $\mathrm{C}^{18} \mathrm{O}$ is enhanced in F1-MW around IRS2, located in the intersection region between F1-M and F1-S. This may be caused by the feedback of IRS1 and IRS2, or by the dynamical interaction between F1-M and F1-S.

\subsection{2. $\mathrm{HCO}^{+}$}

Gaussian fittings were applied pixel-by-pixel to the spectra of $\mathrm{HCO}^{+} J=1-0$, using the Python package PySpecKit ${ }^{10}$. The resulting maps of integrated intensities $(\mathcal{F})$, central velocities $\left(V_{\text {LSR }}\right)$ and line widths $(\Delta V)$ are shown in Fig. 9. The fitted parameters of a pixel are kept only if the pixel itself and at least four of its eight adjacent pixels have SNRs larger than 5. The emission of $\mathrm{HCO}^{+} J=$ $1-0$ around F1-ME overwhelms those around F1$\mathrm{SE}$, in contrast to the case for SO $J_{N}=2_{2}-1_{1}$. The typical line width of $\mathrm{HCO}^{+} J=1-0$ is near $0.6 \mathrm{~km}$ $\mathrm{s}^{-1}$, except for the regions around the four YSOs and the margins of the emission region where the line widths of $\mathrm{HCO}^{+} J=1-0$ can be larger than 1 $\mathrm{km} \mathrm{s}^{-1}$.

We have used the FellWalker (Berry 2015) source extraction algorithm, implemented as part of the Starlink ${ }^{11}$ suite, to identify compact clumps from the $\mathrm{HCO}^{+}$map, following the source extraction processes described in Moore et al. (2015) and Eden et al. (2017). Fourteen $\mathrm{HCO}^{+}$dense clumps were identified, and they are shown in Fig. 2. The peak positions of $\mathrm{HCO}^{+}$and dust clumps are misaligned. $\mathrm{HCO}^{+}$tends to assemble around the junction points of filamentary branches, e.g. H3, H9 and H11-H14. In contrast, the dust emission is more uniformly distributed along the main branch (Sect. 3.2.2) of the filamentary structures (see Fig. 2).

\subsection{3. $\mathrm{HC}_{3} \mathrm{~N}$}

\footnotetext{
${ }^{10} \mathrm{https}: / /$ pythonhosted.org/pyspeckit/

${ }^{11} \mathrm{http} / / /$ starlink.eao.hawaii.edu/starlink/WelcomePage/
}

The $\mathrm{HC}_{3} \mathrm{~N} J=11-10$ and $J=10-9$ intensity maps detected by the NRO $45 \mathrm{~m}$, as well as the rotational temperature map derived from the ratios between the integrated intensities of these two lines are shown in Fig. 10. The enhanced intensity ratios between $\mathrm{HC}_{3} \mathrm{~N} J=11-10$ and $J=10-9$ near IRS 1 may originate from the heating by IRS 1 . The rotational temperatures of $\mathrm{HC}_{3} \mathrm{~N}$ around IRS1 and IRS3 derived from the intensity ratios can be higher than $20 \mathrm{~K}$ (see the lower panel of Fig. 10). Because of the limited SNRs of these two lines, the uncertainties of the derived rotational temperatures can be as high as $5 \mathrm{~K}$. However, it can be confirmed that gas around these YSOs tends to be heated.

The radiation from the YSOs may also play an important role in the evolution of the CCM abundances in the ambient matter around the protostellar sources. In star formation regions, $\mathrm{HC}_{3} \mathrm{~N}$ as well as $\mathrm{HC}_{5} \mathrm{~N}$ would be destroyed by stellar activities (Taniguchi et al. 2018). From Fig. 10, a clue for the erosion of $\mathrm{HC}_{3} \mathrm{~N}$ emission by IRS1 can be seen in the western margin of the clump between IRS1 and IRS2. The enhancements of S-bearing CCMs induced by shocks may also be decomposed by stellar radiation. In evolved stage of SCCC, outflows become weaker while the effects of stellar radiation are getting stronger because of diminishing shielding effects from ambient matter around young stars. This may be the reason why SCCC sources with $X\left[\mathrm{C}_{3} \mathrm{~S}\right]$ exceeding $X\left[\mathrm{HC}_{5} \mathrm{~N}\right]$ are rare.

\section{DISCUSSION}

\subsection{Radial profile of F1-M}

For each of the extracted filaments F1-6 (see Sect. 3.2.1), Gaussian fitting is applied to the radial surface density profile averaged along the main branch (see the right panel of Fig. 11). The fitting to F1 (L1251-A) gives an FWHM radial width of $0.16 \pm 0.02 \mathrm{pc}(1.8 \pm 0.2 \mathrm{arcmin})$, which is smaller than the values of other sub-filaments $(\sim 0.3 \mathrm{pc})$ in this region (see Table 7). For F1 (L1251-A), a width of $0.3 \mathrm{pc}$ was derived by Levshakov et al. (2016) from $\mathrm{NH}_{3}$ emission with an angular reso- 
lution $\sim 40^{\prime \prime}$. This difference can be explained by the higher angular resolutions of the Herschel data $\left(\sim 10^{\prime \prime}\right)$, which resolve more centrally condensed structures near the ridges of filaments. From the right panel of Fig. 11, it is clear that single Gaussian component fits applied to the radial profile of F1 are not as good as for other sub-filaments except for F4. If the radial profile of F1 is fitted with two Gaussian components, the width of the broader one will be consistent with the widths of other substructures with a value $\sim 0.3 \mathrm{pc}$. We have also fitted the radial profile of F1 with a Plummer-like function (Ostriker 1964; Kainulainen et al. 2016)

$$
\Sigma(r)=\Sigma_{0} /\left[1+\left(r / r_{0}\right)^{2}\right]^{(\alpha-1) / 2},
$$

assuming F1 is cylinder-like and oriented parallel to the plane of the sky. The fitted results are shown as red dashed lines in the right panel of Fig. 11. $\mathrm{r}_{0}$ and $\alpha$ are obtained to be $0.05 \mathrm{pc}$ and 2.5 , respectively. The corresponding volume density of $\mathrm{H}_{2}$ and linear density $\left(\mathcal{M}_{l}\right)$ in the central regions are $3 \times 10^{4} \mathrm{~cm}^{-3}$ and $\sim 36 \mathrm{M}_{\odot} \mathrm{pc}^{-1}$, respectively.

The Plummer distribution with $\alpha=4$ describes an isothermal gas cylinder in hydrostatic equilibrium. It gives $n(r) \propto r^{-4}$ when $r \rightarrow \infty$. If $\alpha<2$, the linear density contributed from the mass enclosed in the cylinder with radius of $\mathrm{r}\left(\mathcal{M}_{l}(r)\right)$ is infinite when $r \rightarrow \infty$. Thus a cutoff of radial profile and nonlocal support mechanisms against gravity such as turbulence, magnetism and rotation must be introduced to stabilize the filament. The effective velocity dispersion $\left(\sigma_{1 \mathrm{D}}\right)$ can be expressed as

$\sigma_{1 \mathrm{D}}^{2}=k T / \mu m_{H}+\sigma_{N T}^{2}+v_{A}^{2} /(3-D)+(r \Omega)^{2} /(3-D)$,

where $v_{A}=\sqrt{\frac{B}{4 \pi \rho}}$ is the Alfvén speed, $\sigma_{N T}$ the non-thermal velocity dispersion, $\Omega$ the rotational angular velocity, and $\mathrm{D}$ is the dimension of the considered structures ( 0 for isotropic spheres, 1 for filaments and 2 for sheets). Virial equilibrium requires $\sigma_{1 \mathrm{D}} \propto r^{(1-\alpha / 2)}$ for Plummer distributions with $\alpha<2$. For a Plummer distribution with $\alpha>2$, the linear density $\mathcal{M}_{l}$ is finite, and the requirement of virial equilibrium leads to

$$
\sigma_{1 \mathrm{D}} \rightarrow \sqrt{G \mathcal{M}_{l} / 2} \text { when } r \rightarrow \infty,
$$

where the confined pressure is ignored. For thermal pressure supported filaments, the above formula leads to an expression of the critical linear mass $\left(\mathcal{M}_{l, c}\right)$, i.e.

$$
\mathcal{M}_{l, c}=\frac{2 k \bar{T}}{G \mu m_{H}}
$$

where $\bar{T}$ is the average temperature.

For F1 with $\alpha=2.5$ and $\mathcal{M}_{l} \sim 36 \mathrm{M}_{\odot} \mathrm{pc}^{-1}$, the dynamical equilibrium requires $\sigma_{1 \mathrm{D}} \sim 0.3 \mathrm{~km}$ $\mathrm{s}^{-1}$ which is approximate two times of the sound speed considering $T_{\text {dust }} \sim 10 \mathrm{~K}$ for F1. F1 will be gravitationally unstable if only the thermal pressure is considered, and this is contrary to the result of Levshakov et al. (2016) since the linear density we derived is larger than the value they derived from $\mathrm{NH}_{3}$ lines. The reason may be that the $\mathrm{NH}_{3}$ lines mainly trace the dense subsonic region while the masses derived from the dust continuum are dominated by the supersonic envelopes. The linear mass and Plummer index $(\alpha)$ of F1 are similar to the values $\left(41 \mathrm{M}_{\odot} \mathrm{pc}^{-1}\right.$ and 2.2) for the Serpens filament, which is at the onset of a slightly supercritical collapse (Gong et al. 2018). The $\sigma_{1 \mathrm{D}}$ required to stabilize $\mathrm{F} 1$ is comparable to the observed velocity dispersion $(\delta V=\Delta V / \sqrt{8 \log (2)})$. Overall, F1 is supercritical $\left(\mathrm{M}_{l}>\mathrm{M}_{l, c}\right.$; André et al. 2019) and mainly supported by turbulence. The gradual radial collapse and fragmentations along the ridge tend to change the $\alpha$ from 4 (the value for an isothermal cylinder) to 2 (the value for clumps supported by uniform $\left.\sigma_{1 \mathrm{D}}\right)$.

\subsection{Different filamentary components in L1251-A}

The position-velocity $(\mathrm{P}-\mathrm{V})$ diagrams of $\mathrm{HCO}^{+}$ $J=1-0$ and $\mathrm{SO} 22_{2}-1_{1}$ along the main branch of L1251-A (F1-M) and the side branch (F1-S) are shown in Fig. 12. The velocity patterns have no large scale gradient along F1-S, but show an oscillating pattern along F1-M with the material around 
IRS1 and IRS3 having obviously redder velocities (by $0.2-0.5 \mathrm{~km} \mathrm{~s}^{-1}$ ) than those around the other two YSOs (IRS2 and IRS4) and those around F1$\mathrm{S}$. There is a blue velocity lobe around the position of IRS4 and a red velocity lobe around IRS3 (see Fig. 12). The velocity shifts of the two lobes may originate from outflows and are comparable with the velocity difference between IRS3 and IRS4. The large scale radial and tangential velocity gradients in the main part of L1251-A (Levshakov et al. 2016) may originate from the blend of the two subfilaments.

The length of F1-S is shorter than that of F1-M. The projection of F1-S on the sky plane reaching from the location of $\mathrm{H} 3$, along F1-SE and F1-SW, to the locations of $\mathrm{H} 9 / \mathrm{H} 10$, and further spreading towards H11-H14 (see Fig. 2). IRS1, IRS3 and IRS4 are located on F1-M, but it is not certain whether IRS2 is located on F1-M or F1-S. The F-M is more evolved than F-S, since the emission of dense gas traces such as $\mathrm{N}_{2} \mathrm{H}^{+}$and $\mathrm{HCO}^{+}$ tend to be stronger in F1-M, while tracers of more diffuse gas such as $\mathrm{CO}$ are extensively distributed favoring F1-S. The line widths of $\mathrm{HCO}^{+}$around IRS2 are close to the values along F1-S, and lower than the values around the other three young stellar objects (see lower panel of Fig. 9). Combining the morphologies of dust and molecular line emissions as well as the distribution of central velocities, we speculate that F1-S is closer to us along the line-of-sight with constant velocity, while F1$\mathrm{M}$ is behind F1-S with velocities twisted by forming young stellar objects.

The surface density profile of the dust along the ridge of the main branch (from F3, F1, F5 to F6) is shown in Fig. 11. IRS3 and IRS4 coincide with their nearby local maxima of the dust profile with deviations smaller than $20^{\prime \prime}(0.03 \mathrm{pc})$. The other two YSOs (IRS1 and IRS2) is located at the right and left margins of a dust/ $\mathrm{HCO}^{+}$clump (H8; see Fig. 2) with distances from the center of $\mathrm{H} 8$ of about $1^{\prime}(0.1 \mathrm{pc})$. The dust clumps and the dense gas clumps identified from $\mathrm{HCO}^{+}$emissions are not spatially coincident, but arrange in an alternate pattern (see Fig. 2). The twisted spatial density and velocity distribution along F1-M may be explained by a large-scale MHD-transverse wave originating from outflows of young stellar objects (Nakamura \& Li 2008; Stutz \& Gould 2016; Liu et al. 2019a). The transverse Alfvén wave may also contribute to the assemblage of $\mathrm{HCO}^{+}$around the junction points of different filamentary branches. The gas components should couple well with the magnetic fields and flow sluggishly along the pipelines of magnetic fields. The dust is less affected, with peaks of the dust clumps misaligned with those of the dense gas clumps (see Figs. 2 and 9). Seligman et al. (2019) investigated the non-linear evolution of the magnetized 'resonant drag instabilities' (RDIs), and found the dust organizes into coherent structures and the system exhibits strong dustgas separation. The separation between the gas and dust clumps can also be explained by instabilities induced by magnetic ambipolar diffusion along the filaments (Hosseinirad et al. 2018; Gholipour 2018).

\subsection{Is $C_{3} S$ a unique indicator of SCCC?}

The abundance of sulfur is quite uncertain. However, previous observations show that in cold cores and low mass star formation cores the column densities of $\mathrm{C}_{3} \mathrm{~S}$ are rarely higher than those of $\mathrm{HC}_{5} \mathrm{~N}$. The $\mathrm{C}_{3} \mathrm{~S}$ column densities are even lower than those of $\mathrm{HC}_{7} \mathrm{~N}$ in all starless sources observed in Lupus I (Wu et al. 2019a).

The left panel of Fig. 13 shows the tight correlation between the column density of $\mathrm{C}_{2} \mathrm{~S}$ and $\mathrm{C}_{3} \mathrm{~S}$ in low mass cores quoted from the literatures. Using a conversion factor of 4.3 between $N\left(\mathrm{C}_{2} \mathrm{~S}\right)$ and $N\left(\mathrm{C}_{3} \mathrm{~S}\right)$, the column densities of $\mathrm{C}_{3} \mathrm{~S}$ can be obtained from those of $\mathrm{C}_{2} \mathrm{~S}$ since observations of low level transitions of $\mathrm{C}_{3} \mathrm{~S}$ are rarely reported.

The right panel shows the correlation between the column densities of $\mathrm{C}_{3} \mathrm{~S}$ (derived from $\mathrm{C}_{2} \mathrm{~S}$ ) and $\mathrm{HC}_{5} \mathrm{~N}$ quoted from Suzuki et al. (1992); Hirota et al. (2009). All star forming sources have $N\left(\mathrm{C}_{3} \mathrm{~S}\right)$ lower than $N\left(\mathrm{HC}_{5} \mathrm{~N}\right)$. There is only one 
formerly studied starless sources L1521E having $N\left(\mathrm{C}_{3} \mathrm{~S}\right)>N\left(\mathrm{HC}_{5} \mathrm{~N}\right)$. It seems impossible the cold gas components around young stars are responsible for the abnormally high abundance of $\mathrm{C}_{3} \mathrm{~S}$ detected around YSOs in the L1251 region. Higher abundances of $\mathrm{C}_{3} \mathrm{~S}$ compared to $\mathrm{HC}_{5} \mathrm{~N}$ is a more strict criterion of SCCC in star-forming regions.

\section{SUMMARY}

The previously identified shocked carbon chain chemistry (SCCC) sources all have $N\left(\mathrm{C}_{3} \mathrm{~S}\right)>N\left(\mathrm{HC}_{7} \mathrm{~N}\right)$ but $N\left(\mathrm{C}_{3} \mathrm{~S}\right)<N\left(\mathrm{HC}_{5} \mathrm{~N}\right)$ deduced from Ku-band observations (Wu et al. 2019a). We thus searched for SCCC sources, characterized by $N\left(\mathrm{C}_{3} \mathrm{~S}\right)>N\left(\mathrm{HC}_{5} \mathrm{~N}\right)$, in radio Kband (18-26 GHz) using the Effelsberg $100 \mathrm{~m}$ telescope. We identified L1251-1 as such a source, and L1251-3 as a candidate. L1251-1 is located in L1251-A and harbors a Class Flat young stellar object (IRS1).

We further mapped L1251-A at $3 \mathrm{~mm}$ band using the PMO $13.7 \mathrm{~m}$ telescope and the NRO $45 \mathrm{~m}$ telescope and in CO $J=3-2$ using the JCMT. Combining the obtained spectra and archival data including Spitzer and Herschel continuum maps, we investigated the morphology, environment and dynamical characteristics of L1251-A as well as their relations to the phenomena of SCCC. The main results include:

1. Molecular lines of $\mathrm{NH}_{3}, \mathrm{HC}_{3} \mathrm{~N}, \mathrm{HC}_{5} \mathrm{~N}, \mathrm{C}_{3} \mathrm{~S}$, c- $\mathrm{C}_{3} \mathrm{H}_{2}$ and $\mathrm{C}_{4} \mathrm{H}$ were detected in three sources, L1251-1, L1251-2 and L1251-3. HFS and Gaussian fittings were applied to obtain basic parameters of the detected lines. Column densities and abundances of these detected species were calculated.

2. L1251-1 is characterized by $N\left(\mathrm{C}_{3} \mathrm{~S}\right)$ exceeding $N\left(\mathrm{HC}_{5} \mathrm{~N}\right)$ and is thus a confirmed SCCC source. L1251-3 has a $\mathrm{C}_{3} \mathrm{~S}$ column density marginally larger than that of $\mathrm{HC}_{5} \mathrm{~N}$. L1251-1 has similar abundance ratios of $N\left(\mathrm{C}_{4} \mathrm{H}\right) / N\left(\mathrm{HC}_{3} \mathrm{~N}\right)$ and $N\left(\mathrm{HC}_{3} \mathrm{~N}\right) / N\left(\mathrm{HC}_{5} \mathrm{~N}\right)$ to the SCCC sources in Wu et al. (2019a).
3. The volume densities of the three sources are constrained from the $\mathrm{c}-\mathrm{C}_{3} \mathrm{H}_{2} 1_{1,0}-1_{0,1}$ emission line and the $\mathrm{c}^{-} \mathrm{C}_{3} \mathrm{H}_{2} 2_{2,0}-2_{1,1}$ absorption line based on the RADEX model with an ortho-to-para ratio of c- $\mathrm{C}_{3} \mathrm{H}_{2}$ assumed to be 3 . We find the intensity ratio between $\mathrm{c}-\mathrm{C}_{3} \mathrm{H}_{2} 2_{2,0}-2_{1,1}$ and $\mathrm{c}-\mathrm{C}_{3} \mathrm{H}_{2} 1_{1,0}-1_{0,1}$ (noted as $\mathcal{R}$ ) can serve as a volume density tracer. This method gives a volume density of $3 \times 10^{5} \mathrm{~cm}^{-3}$ for L1251-2, and the values for L1251-1 and L1251-3 are estimated to be $\sim 10^{4} \mathrm{~cm}^{-3}$.

4. The L1251 region consists of hierarchical filaments in Herschel continuum maps. Six sub-filaments labeled as F1 to F6 were extracted using FilFinder. F1 associated with L1251-A has two intertwined branches, the main branch (F1-M) and the side branch (F1-S). F1-S is about $0.4 \mathrm{~km} \mathrm{~s}^{-1}$ bluer than F1-M. Four YSOs (IRS1, IRS2, IRS3 and IRS4) are likely located on F1-M, although there is also possibility that IRS2 is on F1-S.

5. The radial density distribution of $\mathrm{F} 1$ can be well fitted with a Plummer-like function. No large scale velocity gradients, neither radially nor tangentially, are found in the main part of L1251-A.

6. IRS3 and IRS4 (L1251-2) both show strong jets and outflows. A possible shocked region is found in the northwest of IRS4 and we name it IRS4-MNW. IRS4-MNW may be driven by the jet of IRS4. Outflow features are effective in this region and we speculate that there were outflows driven by IRS1 although extinguished now.

7. The interstellar medium around IRS1 and IRS3 has redder velocities than those around the other two YSOs (IRS1 and IRS2) and those on F1-S. The twisted spatial density distribution and velocity distribution along F1-M may present a large-scale MHDtransverse wave, resulting from outflows. 
8. The emission of dense gas tracers such as $\mathrm{HCO}^{+}$and $\mathrm{N}_{2} \mathrm{H}^{+}$is associated with the main branch of F1 (F1-M). However, the emission of $\mathrm{SO}$ and $\mathrm{C}^{18} \mathrm{O}$ is much more enhanced in the eastern part of the side branch of F1 (F1-SE) compared to F1-M. Principal component analysis is applied and confirms this characteristic.

9. The peak positions of $\mathrm{HCO}^{+}$clumps are misaligned with those of the dust clumps.

10. SCCC in L1251-1 may have been caused by outflow activities from the infrared source IRS1. L1251-1 (IRS1) together with the previously identified SCCC source L1251-IRS3 (Wu et al. 2019a) demonstrate that L1251-A is an excellent region to study shocked carbon chain chemistry.

Overall, the outflow activities in L1251-A are responsible for the physical characteristics including the distorted velocity distribution along F1-M, the gas-dust misalignment, and the chemical property related to the abnormal high abundance of $\mathrm{C}_{3} \mathrm{~S}$.

\section{ACKNOWLEDGMENTS}

This project was supported by the National Key R\&D Program of China No. 2017YFA0402600, and the NSFC No. 12033005, 11988101, 1143300811373009,11503035 and 11573036. D.Madones acknowledges support from CONICYT project Basal AFB-170002. Natalia Inostroza acknowledges CONICYT/PCI/REDI170243.

We wish to thank the staff of Effelsberg $100 \mathrm{~m}$, NRO $45 \mathrm{~m}$, PMO $13.7 \mathrm{~m}$ and JCMT $15 \mathrm{~m}$ for their support during the observations. The Effelsberg $100 \mathrm{~m}$ telescope is operated by the MaxPlanck-Institut für Radioastronomie (MPIfR). The Nobeyama Radio Observatory is a branch of the National Astronomical Observatory of Japan, National Institutes of Natural Sciences. The PMO $13.7 \mathrm{~m}$ is operated by the Qinghai station of PMO at Delingha in China. The James Clerk Maxwell Telescope is operated by the East Asian Observatory on behalf of The National Astronomical Observatory of Japan; Academia Sinica Institute of Astronomy and Astrophysics; the Korea Astronomy and Space Science Institute; Center for Astronomical Mega-Science. Additional funding support is provided by the Science and Technology Facilities Council of the United Kingdom and participating universities in the United Kingdom and Canada.

This research has made use of the NASA/IPAC Infrared Science Archive, which is operated by the Jet Propulsion Laboratory, California Institute of Technology, under contract with the National Aeronautics and Space Administration.

Software: NOSTAR, FilFinder (Koch \& Rosolowsky 2015), PySpecKit (Ginsburg \& Mirocha 2011), RADEX (van der Tak et al. 2007), Starlink (Currie et al. 2014), GILDAS/CLASS

\section{REFERENCES}

Agúndez, M., Marcelino, N., Cernicharo, J., Roueff, E., \& Tafalla, M. 2019, A\&A, 625, A147, doi: 10.1051/0004-6361/201935164

André, P., Arzoumanian, D., Könyves, V., Shimajiri, Y., \& Palmeirim, P. 2019, A\&A, 629, L4, doi: 10.1051/0004-6361/201935915
Andre, P., Ward-Thompson, D., \& Barsony, M. 1993, ApJ, 406, 122, doi: 10.1086/172425

André, P., Men'shchikov, A., Bontemps, S., et al. 2010, A\&A, 518, L102, doi: 10.1051/0004-6361/201014666 
Balázs, L. G., Ábrahám, P., Kun, M., Kelemen, J., \& Tóth, L. V. 2004, A\&A, 425, 133, doi: 10.1051/0004-6361:20047059

Benedettini, M., Pezzuto, S., Burton, M. G., et al. 2012, MNRAS, 419, 238, doi: 10.1111/j.1365-2966.2011.19687.x

Berry, D. S. 2015, Astronomy and Computing, 10, 22, doi: 10.1016/j.ascom.2014.11.004

Bianchi, S., Gonçalves, J., Albrecht, M., et al. 2003, A\&A, 399, L43, doi: 10.1051/0004-6361:20030078

Broten, N. W., Oka, T., Avery, L. W., MacLeod, J. M., \& Kroto, H. W. 1978, ApJL, 223, L105, doi: 10.1086/182739

Caselli, P., Benson, P. J., Myers, P. C., \& Tafalla, M. 2002, ApJ, 572, 238, doi: 10.1086/340195

Cohen, M., Wheaton, W. A., \& Megeath, S. T. 2003, AJ, 126, 1090, doi: 10.1086/376474

Cordiner, M. A., Charnley, S. B., Buckle, J. V., Walsh, C., \& Millar, T. J. 2011, ApJL, 730, L18, doi: 10.1088/2041-8205/730/2/L18

Currie, M. J., Berry, D. S., Jenness, T., et al. 2014, in Astronomical Society of the Pacific Conference Series, Vol. 485, Astronomical Data Analysis Software and Systems XXIII, ed. N. Manset \& P. Forshay, 391

Dickens, J. E., Langer, W. D., \& Velusamy, T. 2001, ApJ, 558, 693, doi: 10.1086/322292

Dunham, M. M., Crapsi, A., Evans, Neal J., I., et al. 2008, ApJS, 179, 249, doi: 10.1086/591085

Dunham, M. M., Arce, H. G., Allen, L. E., et al. 2013, AJ, 145, 94, doi: 10.1088/0004-6256/145/4/94

Eden, D. J., Moore, T. J. T., Plume, R., et al. 2017, MNRAS, 469, 2163, doi: 10.1093/mnras/stx874

Enoch, M. L., Evans, II, N. J., Sargent, A. I., \& Glenn, J. 2009, ApJ, 692, 973, doi: 10.1088/0004-637X/692/2/973

Evans, II, N. J., Dunham, M. M., Jørgensen, J. K., et al. 2009, ApJS, 181, 321, doi: 10.1088/0067-0049/181/2/321

Forbrich, J., Tappe, A., Robitaille, T., et al. 2010, ApJ, 716, 1453, doi: 10.1088/0004-637X/716/2/1453

Friesen, R. K., Medeiros, L., Schnee, S., et al. 2013, MNRAS, 436, 1513, doi: 10.1093/mnras/stt1671

Fuente, A., Cernicharo, J., Roueff, E., et al. 2016, A\&A, 593, A94, doi: 10.1051/0004-6361/201628285

Gholipour, M. 2018, ApJ, 864, 108, doi: 10.3847/1538-4357/aad5e3

Ginsburg, A., \& Mirocha, J. 2011, PySpecKit: Python Spectroscopic Toolkit. http://ascl.net/1109.001
Gong, Y., Li, G. X., Mao, R. Q., et al. 2018, A\&A, 620, A62, doi: 10.1051/0004-6361/201833583

Green, G. M., Schlafly, E., Zucker, C., Speagle, J. S., \& Finkbeiner, D. 2019, ApJ, 887, 93, doi: 10.3847/1538-4357/ab5362

Greene, T. P., Wilking, B. A., Andre, P., Young, E. T., \& Lada, C. J. 1994, ApJ, 434, 614, doi: $10.1086 / 174763$

Hartquist, T. W., Dalgarno, A., \& Oppenheimer, M. 1980, ApJ, 236, 182, doi: 10.1086/157731

Hirahara, Y., Suzuki, H., Yamamoto, S., et al. 1992, ApJ, 394, 539, doi: 10.1086/171605

Hirota, T., Maezawa, H., \& Yamamoto, S. 2004, ApJ, 617, 399, doi: 10.1086/425261

Hirota, T., Ohishi, M., \& Yamamoto, S. 2009, ApJ, 699, 585, doi: 10.1088/0004-637X/699/1/585

Hirota, T., \& Yamamoto, S. 2006, ApJ, 646, 258, doi: $10.1086 / 504866$

Ho, P. T. P., \& Townes, C. H. 1983, ARA\&A, 21, 239, doi: 10.1146/annurev.aa.21.090183.001323

Hosseinirad, M., Abbassi, S., Roshan, M., \& Naficy, K. 2018, MNRAS, 475, 2632, doi: $10.1093 / \mathrm{mnras} / \mathrm{stx} 3353$

Kaifu, N., Ohishi, M., Kawaguchi, K., et al. 2004, PASJ, 56, 69, doi: 10.1093/pasj/56.1.69

Kainulainen, J., Hacar, A., Alves, J., et al. 2016, A\&A, 586, A27, doi: 10.1051/0004-6361/201526017

Kamazaki, T., Okumura, S. K., Chikada, Y., et al. 2012, PASJ, 64, 29, doi: 10.1093/pasj/64.2.29

Kauffmann, J., Bertoldi, F., Bourke, T. L., Evans, N. J., I., \& Lee, C. W. 2008, A\&A, 487, 993, doi: 10.1051/0004-6361:200809481

Kauffmann, J., Pillai, T., Shetty, R., Myers, P. C., \& Goodman, A. A. 2010, ApJ, 712, 1137, doi: 10.1088/0004-637X/712/2/1137

Kim, G., Lee, C. W., Maheswar, G., et al. 2019, ApJS, 240, 18, doi: 10.3847/1538-4365/aaf889

Kim, J., Lee, J.-E., Choi, M., et al. 2015, ApJS, 218, 5, doi: 10.1088/0067-0049/218/1/5

Kim, S., Lee, C. W., Gopinathan, M., et al. 2020, ApJ, 891, 169, doi: 10.3847/1538-4357/ab774d

Koch, E. W., \& Rosolowsky, E. W. 2015, MNRAS, 452, 3435, doi: 10.1093/mnras/stv1521

Kroto, H. W., Kirby, C., Walton, D. R. M., et al. 1978, ApJL, 219, L133, doi: 10.1086/182623

Kun, M., \& Prusti, T. 1993, A\&A, 272, 235

Lada, C. J. 1987, in IAU Symposium, Vol. 115, Star Forming Regions, ed. M. Peimbert \& J. Jugaku, 1 
Launhardt, R., Stutz, A. M., Schmiedeke, A., et al. 2013, A\&A, 551, A98, doi: 10.1051/0004-6361/201220477

Law, C. J., Öberg, K. I., Bergner, J. B., \& Graninger, D. 2018, ApJ, 863, 88, doi: $10.3847 / 1538-4357 /$ aacf9d

Lee, J.-E., Lee, H.-G., Shinn, J.-H., et al. 2010, ApJL, 709, L74, doi: 10.1088/2041-8205/709/1/L74

Levshakov, S. A., Henkel, C., Reimers, D., et al. 2013, A\&A, 553, A58, doi: 10.1051/0004-6361/201220354

Levshakov, S. A., Reimers, D., \& Henkel, C. 2016, A\&A, 586, A126, doi: 10.1051/0004-6361/201527783

Liu, H.-L., Stutz, A., \& Yuan, J.-H. 2019a, MNRAS, 487, 1259, doi: 10.1093/mnras/stz1340

Liu, X. C., Wu, Y., Zhang, C., et al. 2019b, Astronomy and Astrophysics, 622, A32, doi: 10.1051/0004-6361/201834411

Loomis, R. A., Burkhardt, A. M., Shingledecker, C. N., et al. 2020, arXiv e-prints, arXiv:2009.11900. https://arxiv.org/abs/2009.11900

Luo, G., Feng, S., Li, D., et al. 2019, ApJ, 885, 82, doi: 10.3847/1538-4357/ab45ef

Lynds, B. T. 1962, ApJS, 7, 1, doi: 10.1086/190072

Madden, S. C., Irvine, W. M., Matthews, H. E., Friberg, P., \& Swade, D. A. 1989, AJ, 97, 1403, doi: $10.1086 / 115081$

Mangum, J. G., \& Shirley, Y. L. 2015, PASP, 127, 266, doi: $10.1086 / 680323$

Matthews, H. E., \& Irvine, W. M. 1985, ApJL, 298, L61, doi: 10.1086/184567

Minamidani, T., Nishimura, A., Miyamoto, Y., et al. 2016, in Society of Photo-Optical Instrumentation Engineers (SPIE) Conference Series, Vol. 9914, Millimeter, Submillimeter, and Far-Infrared Detectors and Instrumentation for Astronomy VIII, ed. W. S. Holland \& J. Zmuidzinas, 99141Z, doi: $10.1117 / 12.2232137$

Moore, T. J. T., Plume, R., Thompson, M. A., et al. 2015, MNRAS, 453, 4264, doi: $10.1093 / \mathrm{mnras} / \mathrm{stv} 1833$

Morris, M., Turner, B. E., Palmer, P., \& Zuckerman, B. 1976, ApJ, 205, 82, doi: 10.1086/154252

Nagy, Z., Spezzano, S., Caselli, P., et al. 2019, A\&A, 630, A136, doi: 10.1051/0004-6361/201935568

Nakamura, F., \& Li, Z.-Y. 2008, ApJ, 687, 354, doi: $10.1086 / 591641$

Ossenkopf, V., \& Henning, T. 1994, A\&A, 291, 943

Ostriker, J. 1964, ApJ, 140, 1056, doi: 10.1086/148005
Ott, M., Witzel, A., Quirrenbach, A., et al. 1994, A\&A, 284, 331

Oya, Y., Sakai, N., Sakai, T., et al. 2014, ApJ, 795, 152, doi: 10.1088/0004-637X/795/2/152

Oyama, T., Ozaki, H., Sumiyoshi, Y., et al. 2020, ApJ, 890, 39, doi: 10.3847/1538-4357/ab6a0a

Park, I. H., Wakelam, V., \& Herbst, E. 2006, A\&A, 449, 631, doi: 10.1051/0004-6361:20054420

Pattle, K., Ward-Thompson, D., Kirk, J. M., et al. 2017, MNRAS, 464, 4255, doi: 10.1093/mnras/stw2648

Robitaille, T. P. 2017, A\&A, 600, A11, doi: 10.1051/0004-6361/201425486

Robitaille, T. P., Whitney, B. A., Indebetouw, R., Wood, K., \& Denzmore, P. 2006, ApJS, 167, 256, doi: $10.1086 / 508424$

Rosvick, J. M., \& Davidge, T. J. 1995, PASP, 107, 49, doi: 10.1086/133514

Sakai, N., Sakai, T., Aikawa, Y., \& Yamamoto, S. 2008a, ApJL, 675, L89, doi: 10.1086/533463

Sakai, N., Sakai, T., Hirota, T., Burton, M., \& Yamamoto, S. 2009, ApJ, 697, 769, doi: 10.1088/0004-637X/697/1/769

Sakai, N., Sakai, T., Hirota, T., \& Yamamoto, S. 2008b, ApJ, 672, 371, doi: 10.1086/523635

Sato, F., \& Fukui, Y. 1989, ApJ, 343, 773, doi: 10.1086/167748

Sato, F., Mizuno, A., Nagahama, T., et al. 1994, ApJ, 435, 279, doi: 10.1086/174813

Schwarz, K. R., Shirley, Y. L., \& Dunham, M. M. 2012, AJ, 144, 115, doi: 10.1088/0004-6256/144/4/115

Seligman, D., Hopkins, P. F., \& Squire, J. 2019, MNRAS, 485, 3991, doi: 10.1093/mnras/stz666

Shan, W., Yang, J., Shi, S., et al. 2012, IEEE Transactions on Terahertz Science and Technology, 2, 593, doi: 10.1109/TTHZ.2012.2213818

Skrutskie, M. F., Cutri, R. M., Stiening, R., et al. 2006, AJ, 131, 1163, doi: 10.1086/498708

Stutz, A. M., \& Gould, A. 2016, A\&A, 590, A2, doi: 10.1051/0004-6361/201527979

Suzuki, H., Yamamoto, S., Ohishi, M., et al. 1992, ApJ, 392, 551, doi: 10.1086/171456

Takakuwa, S., Kawaguchi, K., Mikami, H., \& Saito, M. 2001, PASJ, 53, 251, doi: 10.1093/pasj/53.2.251

Takano, S., Masuda, A., Hirahara, Y., et al. 1998, A\&A, 329, 1156

Taniguchi, K., Herbst, E., Caselli, P., et al. 2019, ApJ, 881, 57, doi: 10.3847/1538-4357/ab2d9e

Taniguchi, K., Ozeki, H., Saito, M., et al. 2016, ApJ, 817, 147, doi: 10.3847/0004-637X/817/2/147 
Taniguchi, K., Saito, M., Sridharan, T. K., \& Minamidani, T. 2018, The Astrophysical Journal, 854, 133, doi: 10.3847/1538-4357/aaa66f

Thaddeus, P., Vrtilek, J. M., \& Gottlieb, C. A. 1985, ApJL, 299, L63, doi: 10.1086/184581

Turner, B. E. 1971, The Astrophysical Journal, 163, L35, doi: 10.1086/180662

van der Tak, F. F. S., Black, J. H., Schöier, F. L., Jansen, D. J., \& van Dishoeck, E. F. 2007, A\&A, 468, 627, doi: 10.1051/0004-6361:20066820

Vastel, C., Quénard, D., Le Gal, R., et al. 2018, MNRAS, 478, 5514, doi: 10.1093/mnras/sty1336

Vrtilek, J. M., Gottlieb, C. A., \& Thaddeus, P. 1987, ApJ, 314, 716, doi: 10.1086/165099

Wilking, B. A., Lada, C. J., \& Young, E. T. 1989, ApJ, 340, 823, doi: 10.1086/167439

Wilson, C. D., Warren, B. E., Israel, F. P., et al. 2009, The Astrophysical Journal, 693, 1736, doi: 10.1088/0004-637X/693/2/1736

Wu, J., Dunham, M. M., Evans, Neal J., I., Bourke, T. L., \& Young, C. H. 2007, AJ, 133, 1560, doi: 10.1086/511959
Wu, Y. 1993, Ap\&SS, 200, 183, doi: 10.1007/BF00627129

Wu, Y., Liu, X., Chen, X., et al. 2019a, Monthly Notices of the Royal Astronomical Society, 488, 495, doi: 10.1093/mnras/stz1498

Wu, Y., Lin, L., Liu, X., et al. 2019b, A\&A, 627, A162, doi: 10.1051/0004-6361/201834184

Yıldız, U. A., Kristensen, L. E., van Dishoeck, E. F., et al. 2015, A\&A, 576, A109, doi: 10.1051/0004-6361/201424538

Yoshida, K., Sakai, N., Tokudome, T., et al. 2015, ApJ, 807, 66, doi: 10.1088/0004-637X/807/1/66

Yuan, J., Wu, Y., Ellingsen, S. P., et al. 2017, ApJS, 231, 11, doi: 10.3847/1538-4365/aa7204

Zhou, S., Wu, Y., Evans, Neal J., I., Fuller, G. A., \& Myers, P. C. 1989, ApJ, 346, 168, doi: 10.1086/167997 
Table 1. The infrared and far-infrared continuum flux (in units of mJy) of YSOs in L1251.

\begin{tabular}{|c|c|c|c|c|c|c|c|c|c|c|c|c|c|c|c|}
\hline YSOs $^{1}$ & RA (J2000) & DEC (J2000) & $\mathrm{J}$ & $\mathrm{H}$ & $\mathrm{K}_{s}$ & $3.6 \mu$ & $4.5 \mu$ & $5.8 \mu$ & $8.0 \mu$ & $24 \mu$ & $70 \mu$ & $160 \mu$ & $350 \mu$ & $850 \mu$ & Class \\
\hline IRS1 (L1251-1) & $22: 29: 33.4$ & $+75: 13: 15.9$ & - & - & - & 5.6 & 10 & 15 & 18 & 55 & 150 & 302 & - & - & Flat \\
\hline IRS2 & 59.5 & & & 1.8 & 6.4 & 15 & 21 & 25 & 27 & 272 & 848 & 1532 & - & 490 & Flat \\
\hline IRS3 & & & - & - & - & 0.13 & 0.42 & & & & 1400 & & & & $0 / \mathrm{I}$ \\
\hline IRS4 (L1251-2) & $22: 31: 05.6$ & $+75: 13: 37.1$ & - & - & - & 0.43 & 1.1 & & 0.37 & 1.9 & 688 & 3310 & 7500 & 1570 & II \\
\hline L1251-3 & $22: 37: 31.13$ & $+75: 10: 41.5$ & - & - & - & 0.06 & 0.08 & 0.035 & 0.248 & 0.82 & 30.1 & 182.1 & - & 1500 & II \\
\hline
\end{tabular}

${ }^{1}$ The data from the $\mathrm{J}(1.25 \mu \mathrm{m}), \mathrm{H}(1.65 \mu \mathrm{m}), \mathrm{K}_{s}(2.16 \mu \mathrm{m})$ bands (Cols. 4-6) are taken from the 2MASS All-Sky Point Source Catalog (PSC) (Skrutskie et al. 2006), and the magnitudes are converted into fluxes using the zero-magnitude attributes described in Cohen et al. (2003). The $3.6 \mu \mathrm{m}, 4.5 \mu \mathrm{m}, 5.8 \mu \mathrm{m}, 8.0 \mu \mathrm{m}$, $24 \mu \mathrm{m}$ and $70 \mu \mathrm{m}$ data (Cols. 7-12) were measured using the instruments IRAC and MIPs on Spitzer space telescope by the c2d team (Evans et al. 2009; Enoch et al. 2009). The $160 \mu \mathrm{m}$ data (Col. 13) are quoted from the PACS Point Source Catalogue in Herschel. The $350 \mu \mathrm{m}$ and $850 \mu \mathrm{m}$ data (Cols 14-15) are obtained from Wu et al. (2007) and Pattle et al. (2017).

Table 2. Observed line and telescope parameters

\begin{tabular}{|c|c|c|c|c|c|c|c|c|c|}
\hline Molecules & Transitions & $\begin{array}{l}\text { Freq. } \\
\mathrm{GHz}\end{array}$ & $\log _{10}\left(\mathrm{~A}_{i j}\right)$ & $\mathrm{S}_{i j}$ & $\begin{array}{c}\mathrm{E}_{u} \\
\mathrm{~K}\end{array}$ & $\mathrm{~g}_{u}$ & $\begin{array}{c}\text { HPBW }^{1} \\
\quad "\end{array}$ & $\begin{array}{c}\Delta_{c h} \\
\mathrm{~km} \mathrm{~s} s^{-1}\end{array}$ & $\begin{array}{c}\mathrm{rms}^{2} \\
\mathrm{mK}\end{array}$ \\
\hline $\mathrm{HC}_{3} \mathrm{~N}$ & $J=2-1, F=3-2$ & 18.196310 & -6.88533 & 0.933 & 1.31 & 7 & $38^{E}$ & 0.63 & $10-60$ \\
\hline $\mathrm{HC}_{3} \mathrm{~N}$ & $J=2-1, F=2-1^{3}$ & 18.196217 & -7.01030 & 0.5 & 1.31 & 5 & $38^{E}$ & 0.63 & \\
\hline $\mathrm{HC}_{5} \mathrm{~N}$ & $J=7-6$ & 18.638611 & -6.18100 & 6.998 & 3.58 & 15 & $38^{E}$ & 0.61 & $10-20$ \\
\hline $\mathrm{HC}_{5} \mathrm{~N}$ & $J=8-7$ & 21.301257 & -6.00337 & 7.997 & 4.60 & 17 & $36^{E}$ & 0.54 & $10-20$ \\
\hline $\mathrm{HC}_{5} \mathrm{~N}$ & $J=9-8$ & 23.963897 & -5.84705 & 8.997 & 5.75 & 19 & $35^{E}$ & 0.48 & $10-20$ \\
\hline $\mathrm{C}_{3} \mathrm{~S}$ & $J=4-3$ & 23.122983 & -6.08154 & 4 & 2.77 & 9 & $35^{E}$ & 0.49 & $10-20$ \\
\hline $\mathrm{NH}_{3}$ & $(1,1)$ & 23.694496 & -6.77534 & 3 & 23.3 & 10 & $35^{E}$ & 0.48 & $10-20$ \\
\hline $\mathrm{NH}_{3}$ & $(2,2)$ & 23.722633 & -6.64906 & 6.664 & 64.4 & 6 & $35^{E}$ & 0.48 & $10-20$ \\
\hline $\mathrm{C}_{4} \mathrm{H}$ & $N=2-1, J=5 / 2-3 / 2, F=3-2$ & 19.015143 & -7.58621 & 2.8 & 1.37 & 7 & $38^{E}$ & 0.6 & $10-20$ \\
\hline c- $\mathrm{C}_{3} \mathrm{H}_{2}$ & $1_{1,0}-1_{0,1}$ & 18.343143 & -6.37404 & 4.50 & 3.23 & 9 & $38^{E}$ & 0.62 & $10-20$ \\
\hline c- $\mathrm{C}_{3} \mathrm{H}_{2}$ & $2,2_{2,0}-2_{1,1}$ & 21.587401 & -6.20187 & 2.28 & 9.71 & 5 & $36^{E}$ & 0.53 & $10-20$ \\
\hline $\mathrm{HCO}^{+}$ & $J=1-0$ & 89.188523 & -4.38103 & 1 & 4.28 & 3 & $19^{N}$ & 0.2 & 60 \\
\hline $\mathrm{SO}$ & $22_{2}-1_{1}$ & 86.093950 & -5.27128 & 1.5 & 19.3 & 5 & $19^{N}$ & 0.2 & 50 \\
\hline $\mathrm{HC}_{3} \mathrm{~N}$ & $J=10-9$ & 90.979023 & -4.23748 & 10 & 24.0 & 63 & $19^{N}$ & 0.2 & 60 \\
\hline $\mathrm{HC}_{3} \mathrm{~N}$ & $J=11-10$ & 100.07639 & -4.11136 & 11 & 28.8 & 69 & $17^{N}$ & 0.2 & 50 \\
\hline \multirow[t]{2}{*}{$\mathrm{C}_{2} \mathrm{H}$} & $N=1-0, J=3 / 2-1 / 2, F=2-1$ & 87.316925 & -5.65605 & 2.4 & 4.2 & 5 & $19^{N}$ & 0.2 & 110 \\
\hline & & & & & & & $56^{P}$ & 0.21 & 55 \\
\hline \multirow[t]{2}{*}{$\mathrm{N}_{2} \mathrm{H}^{+}$} & $J=1-0 F_{1}=2-1$ & 93.173700 & -4.40926 & 5.37 & 4.47 & 15 & $19^{N}$ & 0.2 & 170 \\
\hline & & & & & & & $56^{P}$ & 0.2 & 74 \\
\hline $\mathrm{C}^{18} \mathrm{O}$ & $J=1-0$ & 109.78217 & -7.20302 & 1 & 5.27 & 3 & $15^{N}$ & 0.2 & 150 \\
\hline $\mathrm{CS}$ & $J=2-1$ & 97.980950 & -4.77228 & 2 & 7.05 & 5 & $56^{P}$ & 0.19 & 74 \\
\hline $\mathrm{CO}$ & $J=3-2$ & 345.79598 & -7.20302 & 3 & 31 & 7 & $15^{J}$ & 0.2 & 350 \\
\hline
\end{tabular}

The parameters of listed lines are adopted from splatalogue (http://www.cv.nrao.edu/php/splat/advanced.php).

${ }^{1}$ The superscripts 'E', 'N', 'P', and ' $\mathrm{J}$ ' in column 8 refer to the used facility including the Effelsberg $100 \mathrm{~m}, \mathrm{NRO} 45 \mathrm{~m}$, PMO $13.7 \mathrm{~m}$ and JCMT $15 \mathrm{~m}$ telescope, respectively.

${ }^{2}$ This column shows the 1-sigma rms noise levels on a brightness temperature scale.

${ }^{3} \mathrm{HC}_{3} \mathrm{~N} J=2-1, F=2-1$ and $F=3-2$ remain spectrally unresolved in our observations. 
Table 3. The parameters of the radio K-band lines except ammonia inversion transitions.

\begin{tabular}{lcccccccc}
\hline Transitions & $\mathrm{HC}_{3} \mathrm{~N}$ & $\mathrm{HC}_{5} \mathrm{~N}$ & $\mathrm{HC}_{5} \mathrm{~N}$ & $\mathrm{HC}_{5} \mathrm{~N}$ & $\mathrm{C}_{3} \mathrm{~S}$ & $\mathrm{C}_{4} \mathrm{H}$ & $\mathrm{c}_{-}-\mathrm{C}_{3} \mathrm{H}_{2}$ & $\mathrm{c}-\mathrm{C}_{3} \mathrm{H}_{2}$ \\
& $J=2-1 F=3-2$ & $J=7-6$ & $J=8-7$ & $J=9-8$ & $J=4-3$ & $2_{\frac{5}{2} 3}-2_{\frac{3}{2} 2}$ & $1_{1,0}-1_{0,1}$ & $2_{2,0}-2_{1,1}$ \\
\hline \multirow{7}{*}{ L1251-1 } & $0.36(1)$ & $0.05(1)$ & $0.09(2)$ & $0.13(1)$ & $0.27(4)$ & $0.14(2)$ & $0.71(2)$ & $-0.27(1)$ \\
L1251-2 & $0.65(2)$ & $0.20(1)$ & $0.19(1)$ & $0.32(1)$ & $0.12(1)$ & $0.12(1)$ & $1.34(1)$ & $-0.30(1)$ \\
L1251-3 & $0.22(1)$ & $0.07(1)$ & $0.03(1)$ & $0.11(1)$ & $0.13(1)$ & $0.09(1)$ & $0.48(1)$ & $-0.19(1)$ \\
\hline \multicolumn{7}{c}{ Line widths $\left(\mathrm{km} \mathrm{s}^{-1}\right)^{(1)}$} \\
L1251-1 & $1.3(1)$ & $1.0(2)$ & $1.2(4)$ & $0.9(1)$ & $2.5(5)$ & $1.8(4)$ & $1.4(1)$ & $1.2(1)$ \\
L1251-2 & $1.4(1)$ & $1.4(2)$ & $1.3(1)$ & $1.2(1)$ & $1.0(1)$ & $1.3(2)$ & $1.4(1)$ & $1.3(1)$ \\
L1251-3 & $1.5(2)$ & $1.4(5)$ & $1.1(5)$ & $1.3(2)$ & $1.4(2)$ & $1.9(4)$ & $1.5(1)$ & $1.4(1)$ \\
\hline & & \multicolumn{7}{c}{$V_{L S R}\left(\mathrm{~km} \mathrm{~s}^{-1}\right)$} \\
L1251-1 & $-4.31(4)$ & $-4.4(1)$ & $-4.1(2)$ & $-4.14(6)$ & $-3.7(2)$ & $-4.2(1)$ & $-4.28(2)$ & $-4.14(4)$ \\
L1251-2 & $-4.78(2)$ & $-4.8(1)$ & $-4.7(1)$ & $-4.72(4)$ & $-4.7(1)$ & $-4.7(1)$ & $-4.78(1)$ & $-4.64(3)$ \\
L1251-3 & $-4.19(7)$ & $-4.1(2)$ & $-4.2(3)$ & $-4.02(9)$ & $-3.9(1)$ & $-4.1(2)$ & $-4.12(2)$ & $-3.97(5)$ \\
\hline
\end{tabular}

${ }^{1}$ The values of the line widths and $V_{L S R}$ are not as accurate as the integrated intensities, because of the low velocity resolution mode $\left(\sim 0.5 \mathrm{~km} \mathrm{~s}^{-1}\right)$ chosen for the observations. The errors of line widths and $V_{L S R}$ listed here are given by corresponding HFS and Gaussian fittings.

2 The line widths and $V_{L S R}$ of $\mathrm{HC}_{3} \mathrm{~N}$ are obtained from HFS fittings. The parameters of other lines are obtained from Gaussian fittings. A number in parentheses indicates the uncertainty in the last digit, not accounting for an estimated $10 \%$ uncertainty in amplitude calibration.

Table 4. Parameters derived from HFS fittings of $\mathrm{NH}_{3}(1,1)$ and Gaussian fittings of $\mathrm{NH}_{3}(2,2)$.

\begin{tabular}{lccccc}
\hline source & $\begin{array}{c}\mathrm{v}_{11}{ }^{(1)} \\
\mathrm{km} \mathrm{s}^{-1}\end{array}$ & $\begin{array}{c}\Delta V_{11} \\
\mathrm{~km} \mathrm{~s}^{-1}\end{array}$ & $\begin{array}{c}\mathrm{T}_{M B, 11} \\
\mathrm{~K}\end{array}$ & $\tau_{11}$ & $\begin{array}{c}I_{22}{ }^{(2)} \\
\mathrm{K} \mathrm{km} \mathrm{s}^{-1}\end{array}$ \\
\hline L1251-1 & $-4.15(1)$ & $0.95(1)$ & $1.43(3)$ & $1.72(8)$ & $0.14(2)$ \\
L1251-2 & $-4.64(1)$ & $0.87(1)$ & $3.71(2)$ & $2.59(3)$ & $0.60(1)$ \\
L1251-3 & $-3.95(1)$ & $1.07(3)$ & $0.61(2)$ & $0.6(1)$ & $0.05(1)$ \\
\hline
\end{tabular}

${ }^{1}$ The errors of the $\mathrm{v}_{L S R}$ and $\Delta V$ are mainly contributed by the spectral resolution $\left(\Delta_{r e} \sim 0.5 \mathrm{~km} \mathrm{~s}^{-1}\right)$.

${ }^{2}$ The integrated intensity.

Table 5. The parameters derived from spectra of $\mathrm{NH}_{3}$ and $\mathrm{c}-\mathrm{C}_{3} \mathrm{H}_{2}$, as well as the dust continuum.

\begin{tabular}{ccccccc}
\hline & $T_{\text {ex }}\left(\mathrm{NH}_{3}\right)^{(1)}$ & $T_{\text {rot }}\left(\mathrm{NH}_{3}\right)$ & $T_{\text {dust }}$ & $n^{N H_{3}}\left(\mathrm{H}_{2}\right)$ & $n^{c-C_{3} \mathrm{H}_{2}}\left(\mathrm{H}_{2}\right)$ & $N^{\text {dust }}\left(\mathrm{H}_{2}\right)$ \\
& $\mathrm{K}$ & $\mathrm{K}$ & $\mathrm{K}$ & $10^{4} \mathrm{~cm}^{-3}$ & $10^{4} \mathrm{~cm}^{-3}$ & $10^{22} \mathrm{~cm}^{-2}$ \\
\hline $\mathrm{L} 1251-1$ & 5.0 & $9.6(4)$ & $10.4(3)$ & 1.2 & $\sim 1$ & $1.8(5)$ \\
$\mathrm{L} 1251-2$ & 7.9 & $10.3(2)$ & $10.3(3)$ & 4.1 & 30 & $2.6(8)$ \\
$\mathrm{L} 1251-3$ & 4.4 & $9.7(8)$ & $10.0(3)$ & 0.8 & $\sim 1$ & $1.5(4)$ \\
\hline
\end{tabular}

${ }^{1}$ This should be interpreted as a lower limit of the excitation temperature, considering the unknown beam filling factor. 
Table 6. The column densities.

\begin{tabular}{ccccccc}
\hline Species & $\mathrm{NH}_{3}$ & $\mathrm{HC}_{3} \mathrm{~N}$ & $\mathrm{HC}_{5} \mathrm{~N}$ & $\mathrm{C}_{3} \mathrm{~S}$ & $\mathrm{C}_{4} \mathrm{H}$ & $\mathrm{c}_{-} \mathrm{C}_{3} \mathrm{H}_{2}{ }^{2}$ \\
\cline { 2 - 7 } & $10^{14} \mathrm{~cm}^{-2}$ & $10^{13} \mathrm{~cm}^{-2}$ & $10^{12} \mathrm{~cm}^{-2}$ & $10^{12} \mathrm{~cm}^{-2}$ & $10^{13} \mathrm{~cm}^{-2}$ & $10^{13} \mathrm{~cm}^{-2}$ \\
\hline L1251-1 & $4.1(5)$ & $1.68(9)$ & $2.1(3)$ & $4.6(7)$ & $11(2)$ & $<6.7$ \\
L1251-2 & $8.1(3)$ & $3.0(1)$ & $5.1(3)$ & $2.0(3)$ & $9(1)$ & 1.7 \\
L1251-3 & $1.5(4)$ & $1.02(6)$ & $1.8(3)$ & $2.3(3)$ & $7(1)$ & $<4.8$ \\
\hline \multicolumn{7}{c}{ Abundances } \\
\cline { 2 - 7 } L1251-1 & $2.3(3)$ & $9.3(5)$ & $1.2(2)$ & $2.6(4)$ & $5.6(9)$ & $<3.7$ \\
L1251-2 & $3.1(2)$ & $11.6(5)$ & $2.0(1)$ & $0.8(1)$ & $3.5(4)$ & 0.7 \\
L1251-3 & $1.0(3)$ & $6.8(4)$ & $1.2(2)$ & $1.5(2)$ & $4.0(7)$ & $<3.2$ \\
\hline
\end{tabular}

${ }^{1}$ The uncertainties of column densities are derived from errors of the Gaussian fittings. The uncertainties of the abundances introduced by the uncertainties of $N\left(\mathrm{H}_{2}\right)$ and excitation temperatures are not included.

${ }^{2}$ The column densities and abundances of $\mathrm{c}-\mathrm{C}_{3} \mathrm{H}_{2}$ for L1251-1 and L1251-3 should be taken as upper limits (Sect. 4.2).

Table 7. The basic parameters of the six sub-filaments.

\begin{tabular}{cccccc}
\hline & $\begin{array}{c}L^{1} \\
\mathrm{pc}\end{array}$ & $\begin{array}{c}\Delta^{2} \\
\mathrm{pc}\end{array}$ & $L / \Delta$ & $\begin{array}{c}\mathcal{M}_{l} \\
\mathrm{M}_{\odot} \mathrm{pc}^{-1}\end{array}$ & $\begin{array}{c}\mathrm{M} \\
\mathrm{M}_{\odot}\end{array}$ \\
\hline F1 & $3.33(2)$ & $0.16(1)$ & $21(1)$ & $36(1)$ & $120(3)$ \\
F2 & $1.70(2)$ & $0.30(2)$ & $5.7(4)$ & $34(3)$ & $58(6)$ \\
F3 & $2.29(2)$ & $0.31(1)$ & $7.4(3)$ & $45(2)$ & $100(5)$ \\
F4 & $1.06(2)$ & - & - & - & - \\
F5 & $2.38(2)$ & $0.28(1)$ & $8.5(3)$ & $3.0(1)$ & $7.0(3)$ \\
F6 & $4.46(2)$ & $0.28(1)$ & $11.0(5)$ & $13.0(2)$ & $58(1)$ \\
\hline
\end{tabular}

${ }^{1}$ The length of the main branch. ${ }^{2}$ The fitted radial width. 

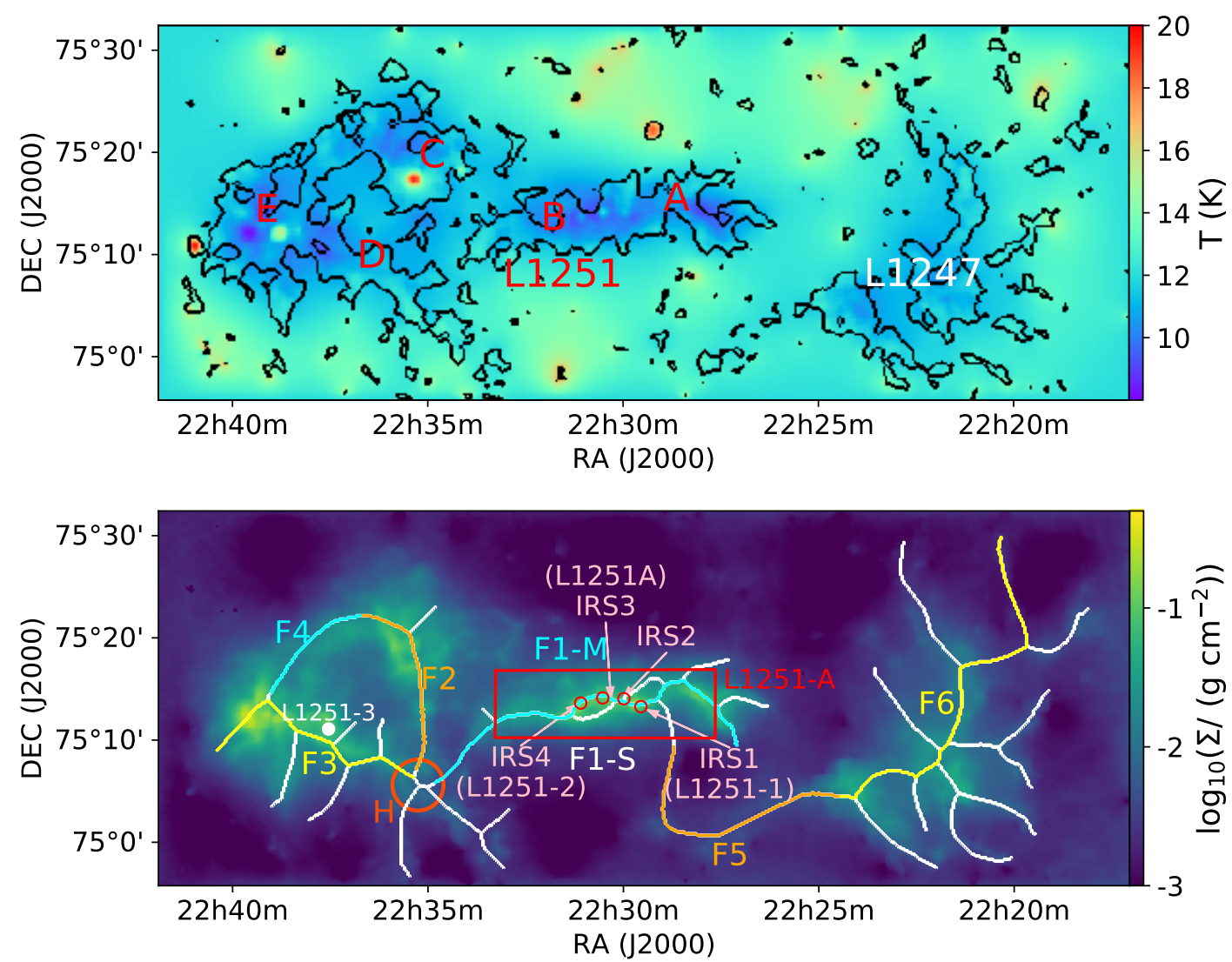

Figure 1. Top: The dust temperatures derived from pixel-by-pixel greybody SED fittings using the $160 \mu \mathrm{m}, 250$ $\mu \mathrm{m}, 350 \mu \mathrm{m}$ and $500 \mu \mathrm{m}$ continuum data from Herschel. Only the pixels within the contours shown in the upper panel were fitted, because the pixels outside have limited SNR in the $160 \mu \mathrm{m}$ band. The dust temperatures outside were extrapolated (see Appendix B). Letters A through E label the positions of the five CO substructures of the L1251 region (Sato et al. 1994). Bottom: The surface density map of the dust. The red box represents our mapped region using PMO $13.7 \mathrm{~m}$ and NRO 45m, and the four YSOs are represented by red open circles. IRS1 and IRS4 are corresponding to L1251-1 and L1251-2. The white filled circle represents L1251-3. The white, cyan, orange, and yellow lines show the skeleton of filamentary structures extracted using FilFinder (Koch \& Rosolowsky 2015). The cyan, orange, and yellow colors are applied to the longest branches (main branches) of the six sub-filaments. The big vermilion circle shows the intersection region of F1, F2 and F3. F1-4 belong to L1251, and F6 is part of L1247. 


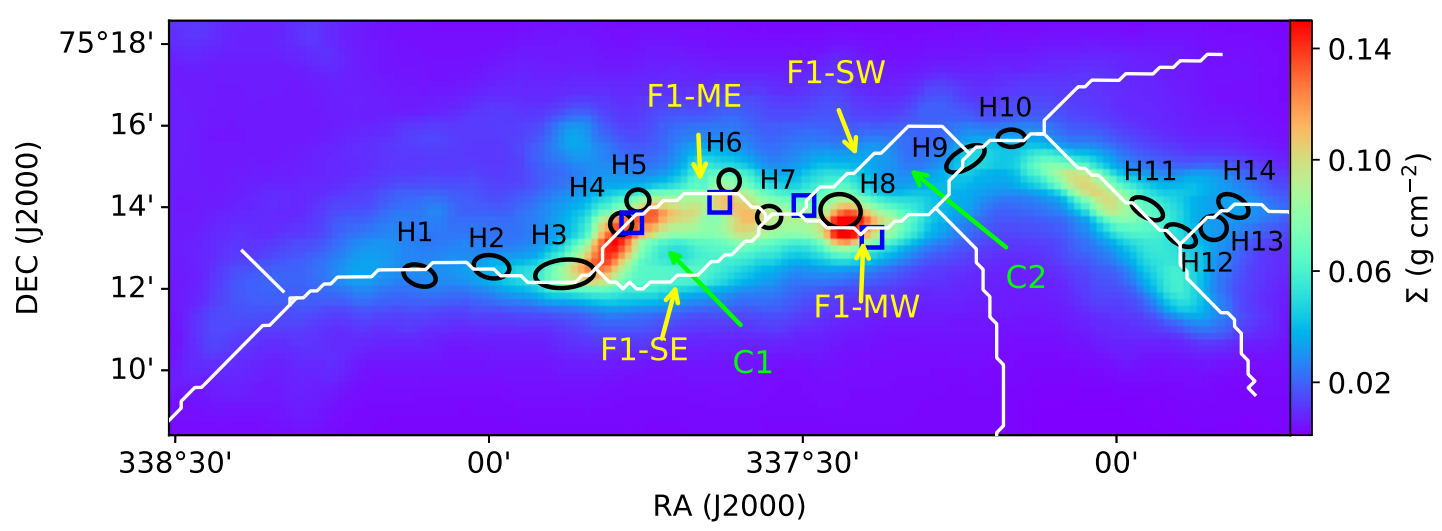

Figure 2. The background shows the surface density of the dust in L1251-A derived from the dust continuum. The black ellipses represent the $\mathrm{HCO}^{+}$clumps identified using the Fellwalker algorithm (see Sect. 5.3). The four YSOs are indicated as blue rectangles. White lines represent the filament skeleton.
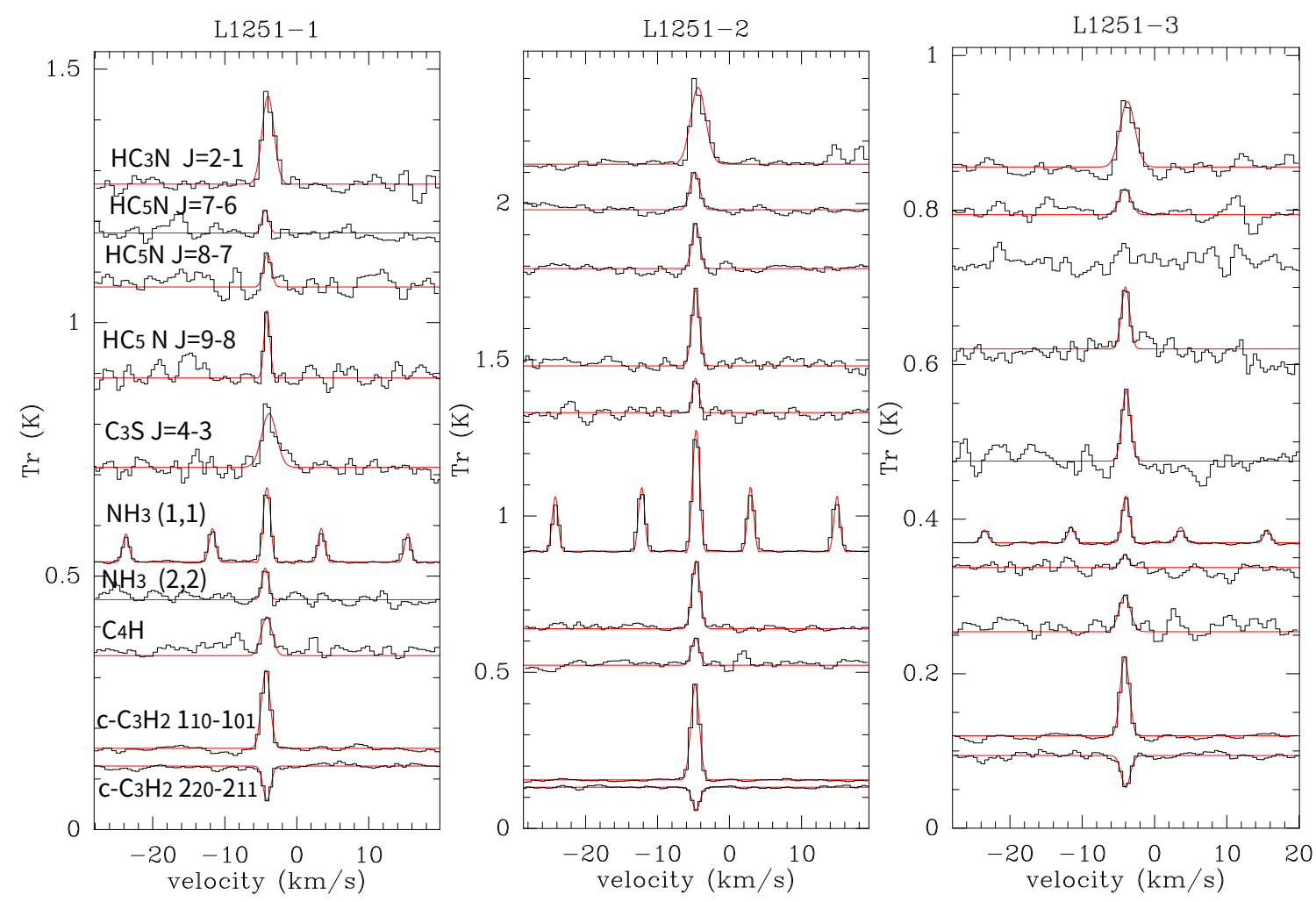

Figure 3. The K-band spectra observed with the Effelsberg $100 \mathrm{~m}$ telescope. For each panel, the spectra from top to bottom are $\mathrm{HC}_{3} \mathrm{~N} J=2-1, \mathrm{HC}_{5} \mathrm{~N} J=7-6,8-7$ and 9-8, $\mathrm{C}_{3} \mathrm{~S} J=4-3, \mathrm{NH}_{3}(1,1), \mathrm{NH}_{3}(2,2), \mathrm{C}_{4} \mathrm{H} N=2-1, J=$ $5 / 2-3 / 2, F=3-2, c-\mathrm{C}_{2} \mathrm{H}_{2} 1_{1,0}-1_{0,1}$ and $\mathrm{c}-\mathrm{C}_{2} \mathrm{H}_{2} 2_{2,0}-2_{1,1}$. The spectra of $\mathrm{NH}_{3}(1,1), \mathrm{NH}_{3}(2,2), \mathrm{c}-\mathrm{C}_{2} \mathrm{H}_{2} 1_{10}-1_{01}$ and c- $\mathrm{C}_{2} \mathrm{H}_{2} 2_{2,0}-2_{1,1}$ have been divided by factors of $10,2,3$ and 3 respectively. 


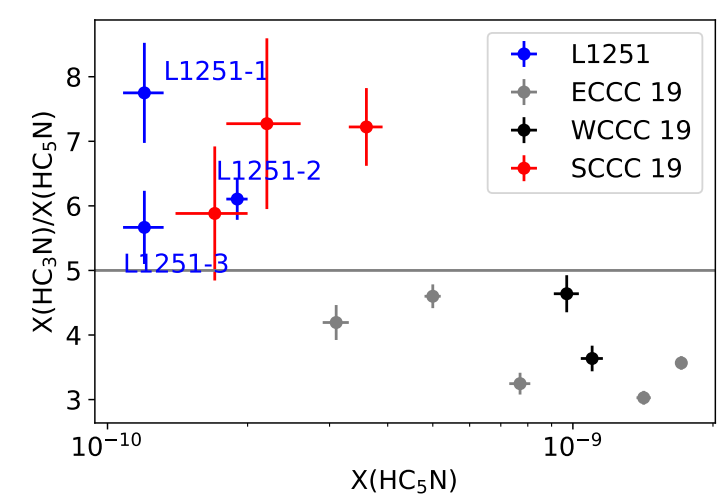

Figure 4. The ratio between the column densities of $\mathrm{HC}_{3} \mathrm{~N}$ and $\mathrm{HC}_{5} \mathrm{~N}$. The three blue points represent L1251-1, L1251-2 and L1251-3. Red and gray points show the data for SCCC sources and early carbon-chain cores (ECCC) from Wu et al. (2019a), detected in Ku band. Black points represent WCCC sources Lupus I-1 ((Sakai et al. 2009; Wu et al. 2019a, IRAS 15398-3359)) and L1489 EMC (Wu et al. 2019b).

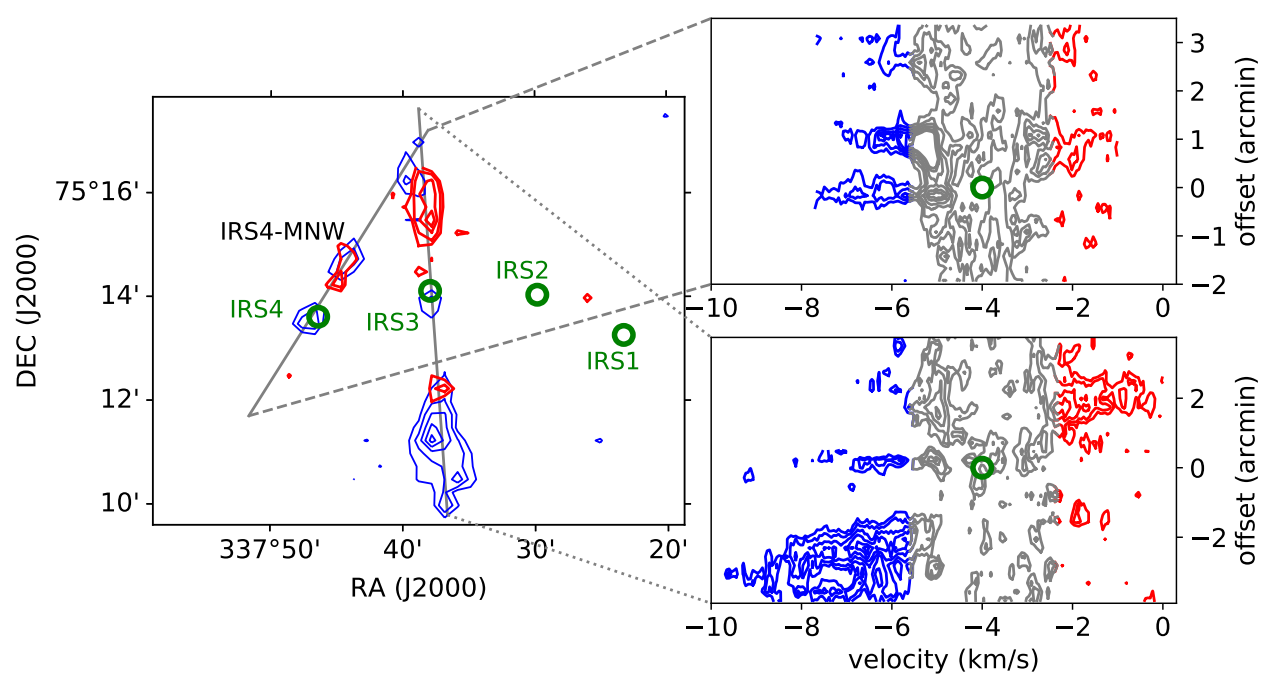

Figure 5. Left: Red and blue contours represent the integrated intensities of the red (from $-2.5 \mathrm{~km} \mathrm{~s}^{-1}$ to $-1 \mathrm{~km} \mathrm{~s}^{-1}$ ) and blue (from $-9 \mathrm{~km} \mathrm{~s}^{-1}$ to $-5.5 \mathrm{~km} \mathrm{~s}^{-1}$ ) wings of the JCMT CO $J=3-2$ lines. Green circles mark the infrared sources IRS1 to IRS4. Right: Position-velocity maps. IRS4 and IRS3 have zero offsets in the upper and lower right panels, respectively. The contour levels are from 25 percent to 85 percent with an increment of 15 percent relative to the maximum intensities. 


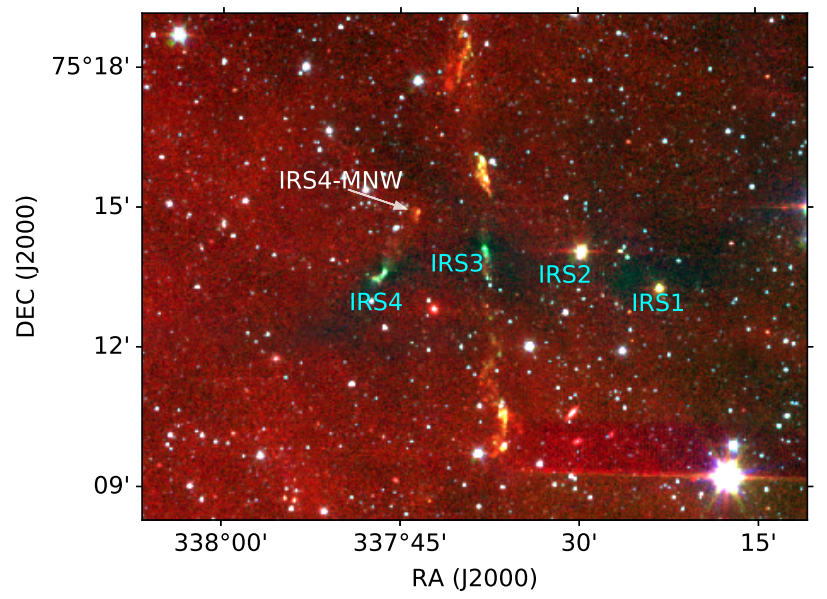

Figure 6. Three-color image comprised of Spitzer IRAC 3.6 (blue), 4.5 (green), and 8.0 (red) $\mu$ m images.

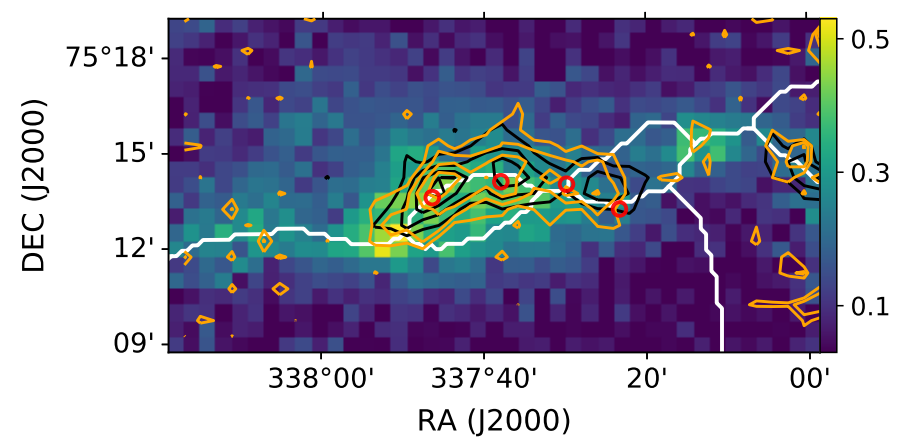

Figure 7. The background, the orange contours and the black contours represent the integrated intensities (in units of $\mathrm{K} \mathrm{km} \mathrm{s}^{-1}$ ) of CS $J=2-1, \mathrm{C}_{2} \mathrm{H} N=2-1, J=3 / 2-1 / 2, F=2-1$ and $\mathrm{N}_{2} \mathrm{H}^{+} J=1-0, F_{1}=2-1$, respectively, mapped by the PMO $13.7 \mathrm{~m}$. The contours have levels from 30 percent to 90 percent and increments of 20 percent, relative to the maximum intensities. The white lines show the skeletons of the filaments extracted from Herschel maps (see Sect. 3.2.1 and Fig. 2). The four YSOs IRS1 to IRS4 are represented by red circles.

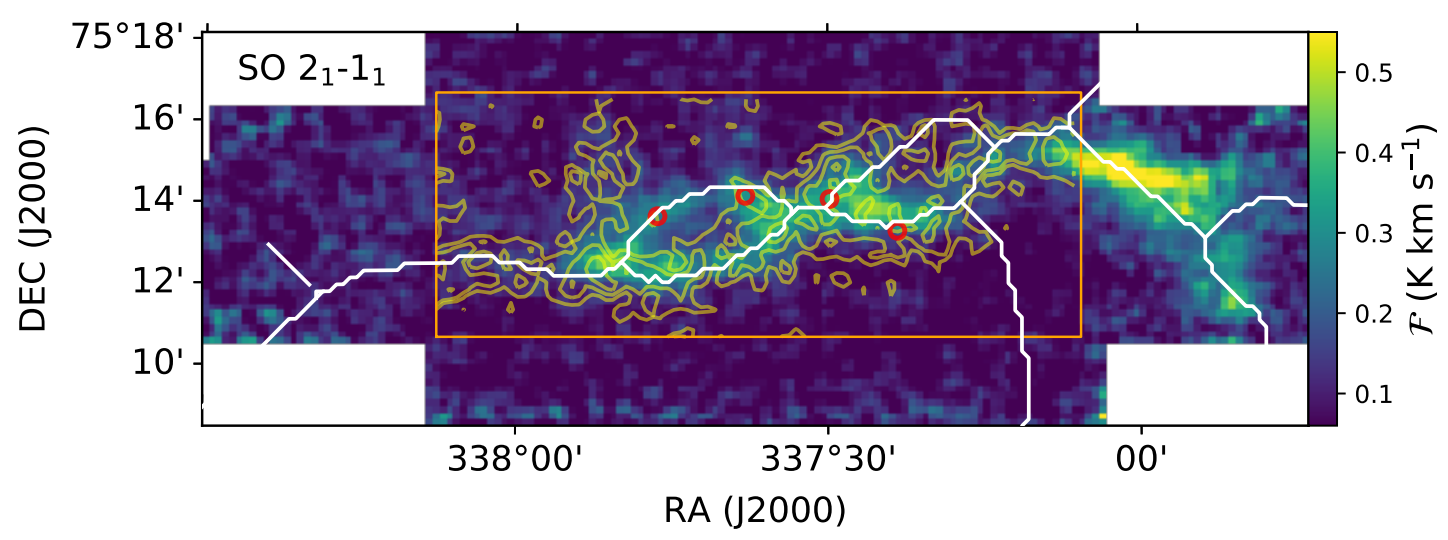

Figure 8. Background is the integrated emission map of SO $2-1$ line observed using the NRO $45 \mathrm{~m}$. Yellow contours show the $\mathrm{C}^{18} \mathrm{O} J=1-0$ emissions of the observed region enclosed in the orange box, with levels from 0.5 to 1.1 and steps of $0.2 \mathrm{~K} \mathrm{~km} \mathrm{~s}^{-1}$. The $\mathrm{C}^{18} \mathrm{O} J=1-0$ lines are integrated within the velocity range $-4.7 \mathrm{~km} \mathrm{~s}^{-1}$ to $-3.5 \mathrm{~km} \mathrm{~s}^{-1}$. See Fig. 7 for the meanings of the white lines and red circles. 


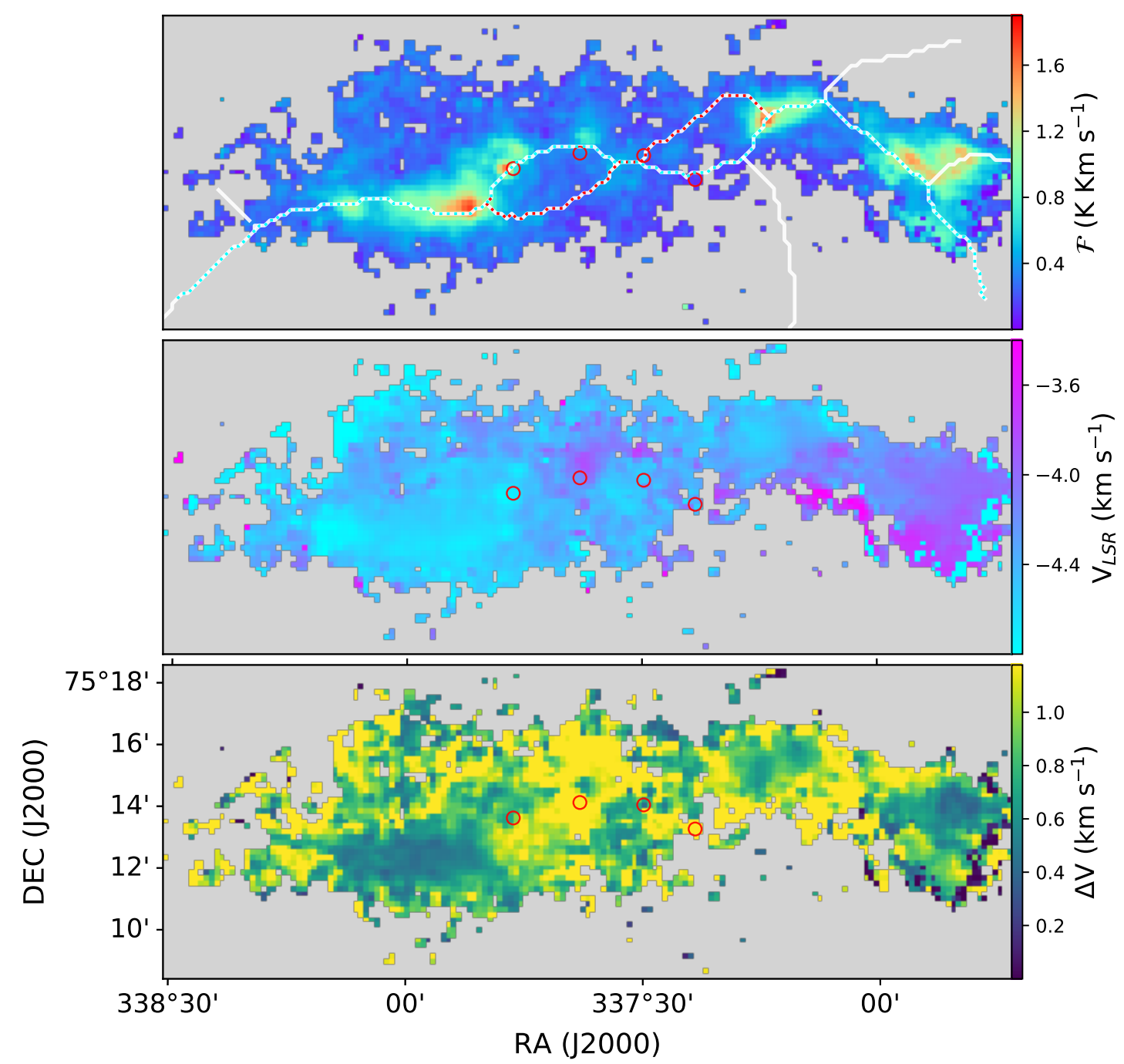

Figure 9. The maps of integrated intensities (top), $V_{\mathrm{LSR}}$ (middle) and line widths $\Delta V$ (bottom) from Gaussian fittings applied to $\mathrm{HCO}^{+} J=1-0$ observed using the NRO $45 \mathrm{~m}$. In the top panel, white and cyan lines show the skeleton of F1. The cyan line represents the main branch of F1. The dotted red line shows the side branch (F1-S) intertwined with the main branch. The four YSOs IRS1 to IRS4 are represented by red circles. 

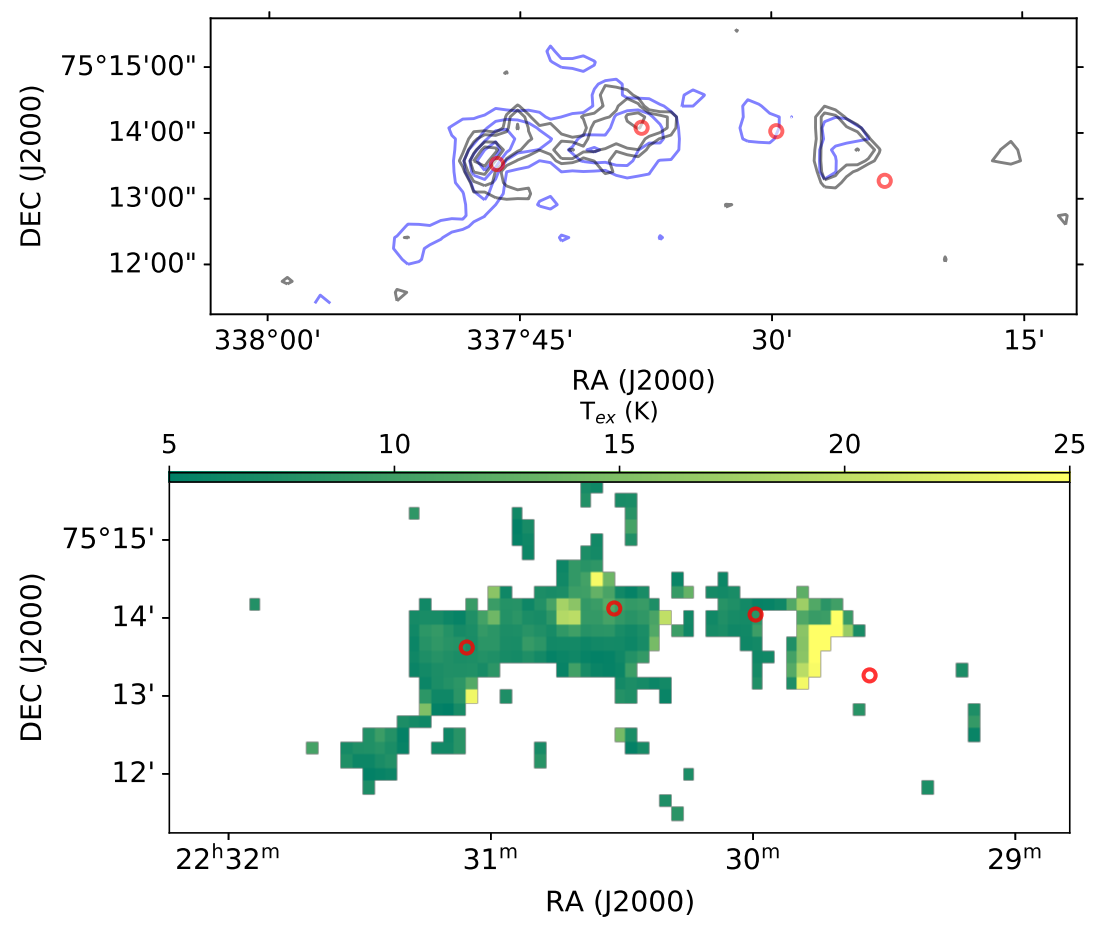

Figure 10. Upper panel: The blue contours show the integrated intensities of $\mathrm{HC}_{3} \mathrm{~N} J=10-9$ observed using the NRO $45 \mathrm{~m}$, with levels from 0.1 to 0.35 and increments of $0.05 \mathrm{~K} \mathrm{~km} \mathrm{~s}^{-1}$. The black contours show the integrated intensities of $\mathrm{HC}_{3} \mathrm{~N} J=11-10$ observed using the $\mathrm{NRO} 45 \mathrm{~m}$, with levels from 0.06 to 0.18 and steps of $0.04 \mathrm{~K} \mathrm{~km}$ $\mathrm{s}^{-1}$. Lower panel: The excitation temperatures derived from the ratios between the integrated intensities of $\mathrm{HC}_{3} \mathrm{~N} J=$ 11-10 and $\mathrm{HC}_{3} \mathrm{~N} J=10-9$. The four YSOs IRS1 to IRS4 are represented by red circles.
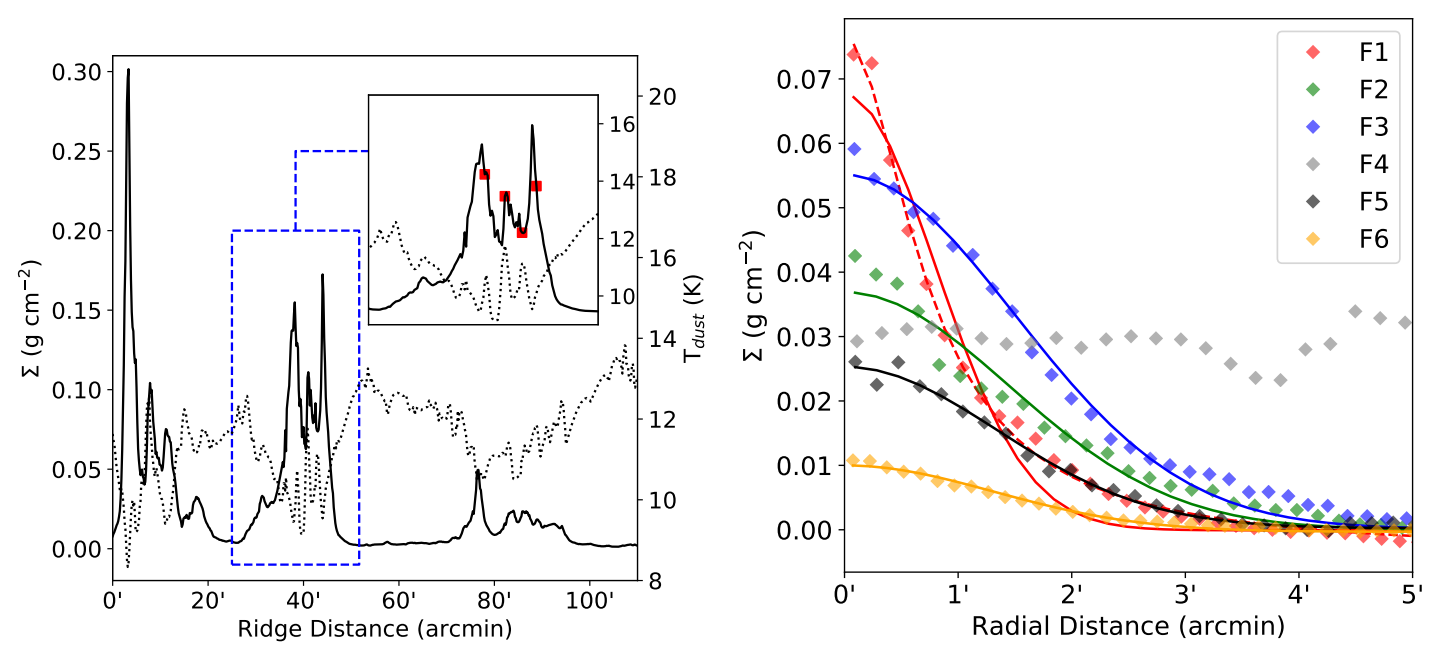

Figure 11. Left: The profiles of surface density and dust temperature along the longest branch of the whole filamentary structure shown in Fig. 1, which is stitched from the main branches of the sub-filaments F3, F1, F5 and F6. The profiles enclosed in the dashed blue box, corresponding to F2, are zoomed in and shown at the top-right corner, with the red filled squares showing the locations of the four YSOs. Right: The averaged radial surface density profiles of sub-filaments, with Gaussian fittings except for F4. The surface densities of F5 have been multiplied by a factor of ten. 


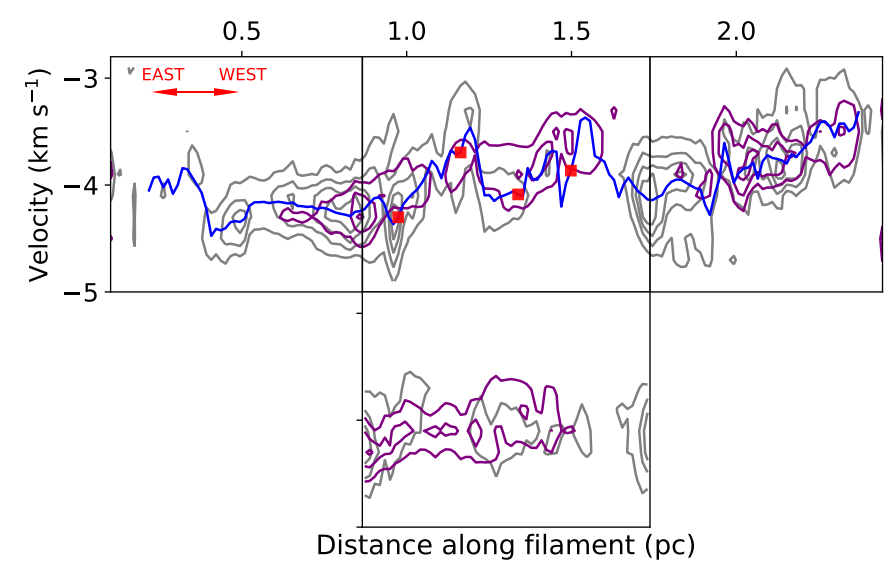

Figure 12. The P-V diagram of $\mathrm{HCO}^{+} J=1-0$ (gray contours) and $\mathrm{SO} J_{N}=2_{2}-1_{1}$ (purple contours) along F1-M (upper panel) and F1-S (lower panel). Blue lines show the fitted central velocity of $\mathrm{HCO}^{+} J=1-0$, and the red filled squares represent the locations of the four YSOs (IRS1 to IRS4 from right to left).
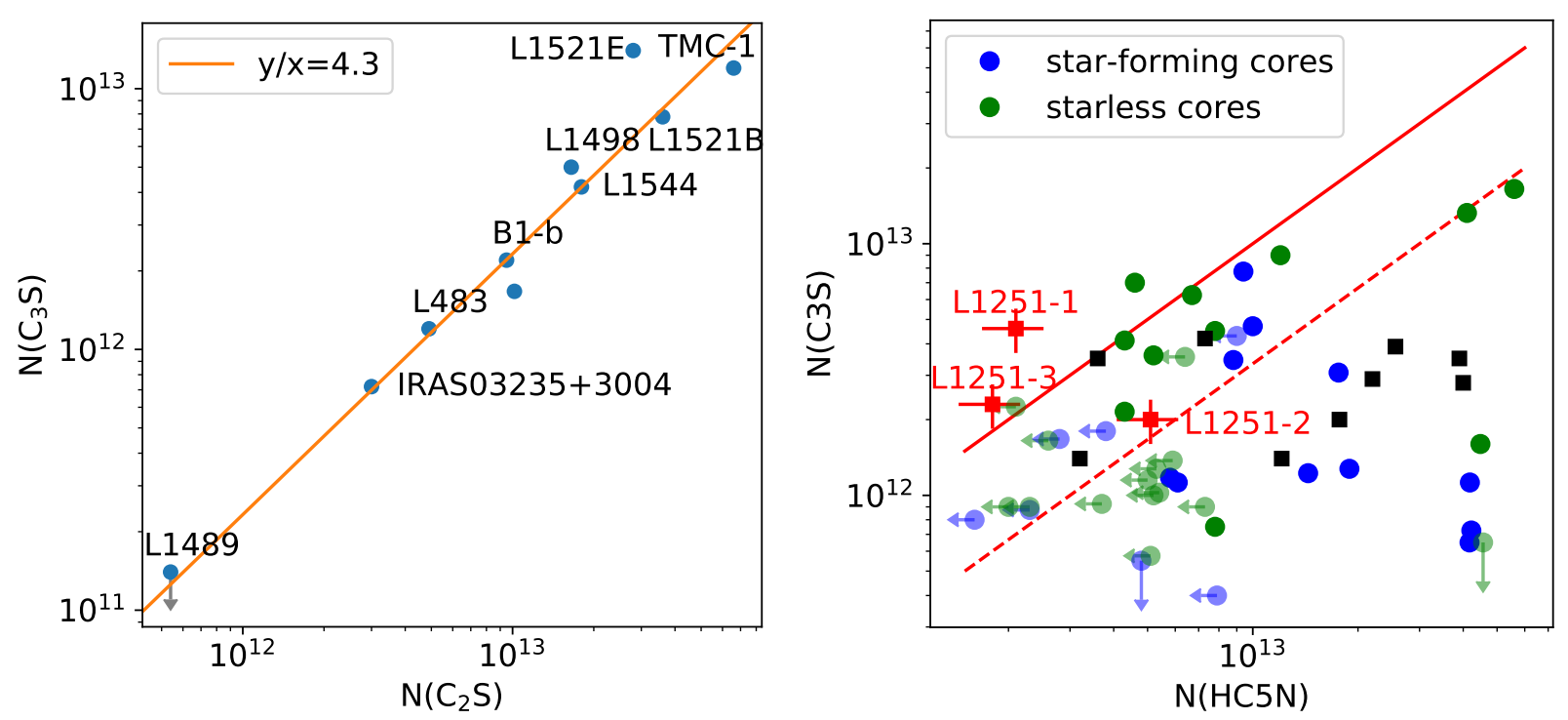

Figure 13. Left: the correlation between the column densities of $\mathrm{C}_{2} \mathrm{~S}$ and $\mathrm{C}_{3} \mathrm{~S}$ of low mass cores quoted from the literature (Hirota et al. 2004; Hirota \& Yamamoto 2006; Law et al. 2018; Agúndez et al. 2019; Nagy et al. 2019). Right: the correlation between the column densities of $\mathrm{C}_{3} \mathrm{~S}$ (derived from $\mathrm{C}_{2} \mathrm{~S}$ ) and $\mathrm{HC}_{5} \mathrm{~N}$ quoted from Suzuki et al. (1992); Hirota et al. (2009). The black filled squares represent sources detected in Wu et al. (2019a). The solid and dashed red lines represent $y=x$ and $y=x / 3$, respectively. 


\section{APPENDIX}

\section{A. YSO CLASSFICATION}

YSO classfication based on the spectral index $\left(\alpha_{\mathrm{IR}}\right)$ in near- and mid-infrared bands defined as $\alpha_{\mathrm{IR}}=d\left(\lambda L_{\lambda}\right) / d \lambda$ was first proposed by Lada (1987), and followed and improved by works such as Wilking et al. (1989) and Greene et al. (1994). The four class system for low mass YSOs based on $\alpha_{\mathrm{IR}}$ commonly used today can be described as

$$
\begin{aligned}
& \text { I } \alpha_{\mathrm{IR}} \geq 0.3 \\
& \text { Flat }-0.3 \leq \alpha_{\mathrm{IR}}<0.3 \\
& \text { II }-1.6 \leq \alpha_{\mathrm{IR}}<-0.3 \\
& \text { III } \alpha_{\mathrm{IR}} \leq-1.6
\end{aligned}
$$

Andre et al. (1993) suggested a class "before" Class I named Class 0, and the YSOs located between Class 0 and Class I have protostellar masses similar to that of the remaining envelope. IRS1, IRS2, IRS3 and IRS4 have spectral indices of 0.19 , $0.33,0.70$ and -0.52 , respectively (Evans et al. 2009). Robitaille et al. (2006) found that YSOs with different spectral classes are distributed in different regions in the [3.6]-[5.8] v.s. [8.0]-[24] color-color diagram, and can also be used to classify YSOs. The four YSOs in the L1251-A region are all formerly located in the Class $0 / \mathrm{I}$ regions. IRS2 was re-classified by Dunham et al. (2013) from Class I to Class Flat through de-reddening it using an extinction value from the literatures.

We fitted the spectral energy distribution (SED) of these four YSOs using the model of Robitaille (2017). Since our fitted sources except IRS2 do not have data in 2MASS bands, the parameters of central stars and disks can not be well constrained. The upper limits adopted as one tenth the values of IRS2 are fed to the fitting procedures, instead. The distances are fixed at $300 \mathrm{pc}$, the V-band extinctions $\left(\mathrm{A}_{V}\right)$ are limited in the range of 0-50 mag, and the extinction law we adopted is the same as that used in Forbrich et al. (2010). The fitted luminosities of central stars $\left(L_{\star}\right)$ of IRS1, IRS2, IRS3 and IRS4 are $0.3 L_{\odot}, 1.4 L_{\odot}, 1.1 L_{\odot}$ and $0.25 L_{\odot}$, respectively. If $A_{V}>40 \mathrm{mag}$ is permitted for IRS3 (Lee et al. 2010), corresponding to a deeply embedded young stellar object with a high accretion rate, the unobscured $L_{\star}$ can be much higher than the value listed here.

All arguments considered, the IRS3 is a Class 0/I object, IRS4 is classified as Class II, and IRS1 and IRS2 are classified as Class Flat.

\section{B. GREYBODY SED FITTING}

Pixel-by-pixel greybody SED fittings are applied to the Herschel archive data ${ }^{12}$ covering the L1251 region in the 160, 250, 350 and $500 \mu \mathrm{m}$ bands, observed by André et al. (2010) as part of the Herschel Gould Belt Survey. The Herschel $70 \mu \mathrm{m}$ data were not used in our fittings, because of limited SNR in this band. We first filtered out the background emissions of each map following Yuan et al. (2017), using the CUPID - findback algorithm of the Starlink suite. Then, we convolve each Herschel map with a Gaussian Kernel with FWHM (in unit arcsec)

$$
\sqrt{35.2^{2}-\theta_{\lambda}^{2}}
$$

where $\theta_{\lambda}$ is the HPBW of the convolved Herschel map, and 35.2 (in arcsec) is the HPBW of the Herschel $500 \mu \mathrm{m}$ map. The convolved maps were then re-gridded to obtain aligned images with spatial pixel size $10^{\prime \prime} \times 10^{\prime \prime}$. For each pixel, its corresponding intensities at different wavelengths in the range of $160-500 \mu \mathrm{m}$ are fitted with a blackbody model

$$
I_{v}=B_{v}\left(T_{\text {dust }}\right)\left(1-e^{-\tau_{v}}\right)
$$

where $B_{v}$ is the Planck function, and the dust optical depth $\tau_{v}$ can be expressed as

$$
\tau_{v}=\Sigma \cdot \kappa_{v} / R_{g d}
$$

\footnotetext{
${ }^{12}$ http://archives.esac.esa.int/hsa/whsa/
} 
Here, $\Sigma$ is the surface density, and $R_{g d}$ is the gasto-dust ratio which is assumed to be 100 . The dust opacity $\kappa_{v}$ can be expressed as a power law in frequency,

$$
\kappa_{v}=\kappa(600 G H z)\left(\frac{v}{600 G H z}\right)^{\beta} .
$$

The reference dust opacity at $600 \mathrm{GHz}(500 \mu \mathrm{m})$ can be adopted as $5 \mathrm{~cm}^{2} \mathrm{~g}^{-1}$, the value for coagulated grains with thin ice mantles given by Ossenkopf \& Henning (1994) (hereafter OH5). In this work, $\kappa(600 \mathrm{GHz})=3.33 \mathrm{~cm}^{2} \mathrm{~g}^{-1}$ was adopted, which is scaled down by a factor of 1.5 from the OH5 value as suggested by Bianchi et al. (2003); Kauffmann et al. (2010). $\beta$ is fixed at 2. There are two free parameters, the dust temperature $T_{\text {dust }}$ and the surface density $\Sigma$ to be obtained from the fitting procedures.

The uncertainty of the intensity at pixel $(i, j)$ was taken as $\sigma_{v ; i, j}=\sqrt{\left(\sigma_{v}^{a b s}\right)^{2}+\left(\sigma_{v ; i, j}^{r e l}\right)^{2}}$, where $\sigma_{v}^{a b s}$ was estimated as the rms value of regions free from emission, while $\sigma_{v ; i, j}^{r e l}$ is taken as 15 percent of the intensity of that pixel, based on the report by Launhardt et al. (2013). Only the pixels with intensities larger than $3 \sigma_{v ; i, j}$ at all four bands were fitted by minimizing

$$
\chi_{i, j}^{2}=\sum_{v}\left(I_{v ; i j}\left(T_{i j}, \Sigma_{i j}\right)-I_{v ; i, j}^{\text {Herschel }}\right)^{2} / \sigma_{v ; i, j}^{2}
$$

The fitted maps of $T_{\text {dust }}$ and $\Sigma$ are shown in Fig. 1 .

The fitted pixels are enclosed by the black contour shown in the upper panel of Fig. 1. The dust temperatures are as low as about $8 \mathrm{~K}$ in the central part of the fitted regions, and increase to about $12 \mathrm{~K}$ at the margins. For pixels outside the fitted regions, the dust temperatures are extrapolated by solving the Laplace's equation $\nabla^{2} T=0$, with the values of the fitted regions unchanged and the values of the pixels at the margins of the whole map fixed as $12 \mathrm{~K}$. Numerically, $\nabla^{2} T=0$ can be solved by iterating

$$
T_{i, j}^{n+1}= \begin{cases}\frac{1}{4} \sum_{\Delta j \in\{-1,1\}} \sum_{\Delta i \in\{-1,1\}} T_{i+\Delta i, j+\Delta j}^{n} & \text { for }(i, j) \notin C \\ T_{i, j}^{n} & \text { for }(i, j) \in C\end{cases}
$$

where $C$ is the set consisting of the fitted pixels and the marginal pixels of the whole map. The surface densities for pixels outside the fitted regions are calculated based on the $500 \mu \mathrm{m}$ intensities through equation B1 and B3, and the extrapolated dust temperatures.

\section{CALCULATE COLUMN DENSITIES}

The column densities of molecules can be derived through (Wilson et al. 2009; Mangum \& Shirley 2015)

$N=\frac{3 k}{8 \pi^{3} v} \frac{Q_{\mathrm{rot}} \exp \left(E_{u} / k T_{\mathrm{ex}}\right)}{S \mu^{2}} \frac{J\left(T_{\mathrm{ex}}\right)}{J\left(T_{\mathrm{ex}}\right)-J\left(T_{\mathrm{bg}}\right)} \int T_{r} d v$

where $\mathrm{h}$ is Planck's constant, $\mathrm{c}$ is the speed of light, $k$ is the Boltzmann constant, $\mathrm{S}$ is the line strength, $\mu$ is the dipole moment, and $Q_{\text {rot }}$ the partition function.

For $\mathrm{NH}_{3}(1,1)$, the optical depth $(\tau)$, the intrinsic full-width at half power linewidth $\left(\Delta V_{\text {in }}\right)$, the LSR velocity $\left(V_{\text {LSR }}\right)$ and the amplitude $\mathcal{A}$ can be obtained through hyperfine structure (HFS) fitting $T_{b}(v)=\mathcal{A}\left(1-e^{-\tau(v)}\right)$ with

$$
\tau(v)=\tau_{\text {tot }} \sum_{0}^{N-1} r_{i} \times \exp \left(-4 \ln (2)\left(\frac{v-v_{\mathrm{LSR}}-v_{i}}{\Delta V_{\mathrm{in}}}\right)^{2}\right)
$$

where where the $r_{i}$ are the relative intensities of the individual hyerfine (hf) lines in the optically thin case under conditions of thermodynamical equilibrium. The excitation temperature $\left(T_{\mathrm{ex}}\right)$ can be obtained from

$$
\mathcal{A}=\eta\left(J_{v}\left(T_{\text {ex }}\right)-J_{v}\left(T_{\text {bg }}\right)\right)
$$

where $J_{v}(T)$ is defined by $J_{v}(T)=\frac{h v / k}{e^{h v / k T}-1}$, and $T_{\mathrm{bg}}$ is the background temperature. 
For $\mathrm{NH}_{3}$, the equation to calculate the column densities of molecules in the $(1,1)$ and $(2,2)$ states can be expressed as

$$
N_{J K}=\zeta_{J K} \mathcal{A} \tau_{\text {tot }} \Delta V_{\text {in }}\left(\frac{1}{\eta}+\frac{J_{\mathrm{bg}}+0.5 h v / k}{\mathcal{A}}\right)
$$

with $\zeta_{11}=1.3850 \times 10^{13}, \zeta_{22}=1.0375 \times 10^{13}(\mathrm{Lev}-$ shakov et al. 2013). Rotational temperatures $\left(T_{\text {rot }}^{21}\right)$ can also be derived from the population ratio between the $(2,2)$ and $(1,1)$ states of $\mathrm{NH}_{3}$.

The rotational temperature $T_{\text {rot }}^{21}$ is adopted to calculate the total column density of $\mathrm{NH}_{3}\left(N\left(\mathrm{NH}_{3}\right)\right)$ assuming only the metastable states are effectively populated,

$N\left(\mathrm{NH}_{3}\right)=N_{11} \sum_{i=0} \frac{g_{I ; K=i}}{g_{I ; K=1}} \frac{2 i+1}{3} \exp \left(\frac{-\left(E_{i}-23.2\right)}{T_{\text {rot }}}\right)$

in which the ratio between the nuclear spin degeneracy $\left(g_{I}\right)$ of para- $\mathrm{NH}_{3}(K \neq 3 n)$ and that of ortho$\mathrm{NH}_{3}(K=3 n)$ is two.

The volume density can be estimated for $\mathrm{T}_{\mathrm{ex}} \neq \mathrm{T}_{\text {kin }}$ through (Ho \& Townes 1983; Levshakov et al. 2013)

$$
n\left(\mathrm{H}_{2}\right)=\frac{A}{C}\left[\frac{J_{v}\left(T_{\mathrm{ex}}-J_{v}\left(T_{\mathrm{bg}}\right)\right)}{J_{v}\left(T_{\mathrm{kin}}\right)-J_{v}\left(T_{\mathrm{ex}}\right)}\right]\left[1+\frac{J_{v}\left(T_{\mathrm{kin}}\right)}{h v / k}\right]
$$

where $A$ is the Einstein A-coefficient and $C$ is the rate coefficient for collisional de-excitation.

\section{EMISSION MODEL OF C- $\mathrm{C}_{3} \mathrm{H}_{2}$}

We calculated the intensities of the $\mathrm{c}-\mathrm{C}_{3} \mathrm{H}_{2} 1_{1,0}-$ $1_{0,1}$ and $\mathrm{c}-\mathrm{C}_{3} \mathrm{H}_{2} \quad 2_{2,0}-2_{1,1}$ lines produced in a uniform medium using the non-LTE radiative transfer code RADEX (van der Tak et al. 2007). The free parameters are the column density of para$\mathrm{C}_{3} \mathrm{H}_{2}\left(N^{p}\right)$, the kinetic temperature $\left(T_{\text {kin }}\right)$, as well as the densities of the collision partners. In our cases, $\mathrm{H}_{2}$ is taken as the only collision partner for both ortho- $\mathrm{C}_{3} \mathrm{H}_{2}$ and para $-\mathrm{C}_{3} \mathrm{H}_{2}$, and the background emission is fixed as $2.73 \mathrm{~K}$. The space of the free input parameters $\left(n\left(\mathrm{H}_{2}\right), N^{p}, T_{\mathrm{kin}}\right)$ is gridded with $n\left(\mathrm{H}_{2}\right)$ and $N(\mathrm{X})$ evenly sampled on a $\log$ scale stepped by a factor of three from $10^{3}$ to $10^{7}$ $\mathrm{cm}^{-3}$ and from $10^{11}$ to $10^{15} \mathrm{~cm}^{-2}$ respectively, and $T_{\text {kin }}$ evenly sampled from 5 to $30 \mathrm{~K}$ stepped by $1 \mathrm{~K}$. For each set of input parameter, the integrated intensities (in units of $\mathrm{K} \mathrm{km} \mathrm{s}^{-1}$ ) of c- $\mathrm{C}_{3} \mathrm{H}_{2} 1_{1,0}-1_{0,1}$ $\left(\mathcal{F}^{o}\right)$ and that of $\mathrm{c}-\mathrm{C}_{3} \mathrm{H}_{2} 2_{2,0}-2_{1,1}\left(\mathcal{F}^{p}\right)$ were calculated. Via extrapolation, the $3 \mathrm{D}$ surfaces $\mathcal{F}^{p}\left(n\left(\mathrm{H}_{2}\right)\right.$, $\left.N^{p}, T_{\text {kin }}\right)$ and $\mathcal{F}^{o}\left(n\left(\mathrm{H}_{2}\right), N^{o}, T_{\text {kin }}\right)$ can be obtained. Since the $\mathrm{o} / \mathrm{p}$ ratios are fixed at three, the flux ratio between $\mathrm{c}-\mathrm{C}_{3} \mathrm{H}_{2} \quad 1_{1,0}-1_{0,1}$ and $2_{2,0}-2_{1,1}(\mathcal{R})$ can be expressed as

$$
\mathcal{R}\left(n\left(\mathrm{H}_{2}\right), N^{p}, T_{\text {kin }}\right)=\frac{\mathcal{F}^{p}\left(n\left(\mathrm{H}_{2}\right), N^{p}, T_{\text {kin }}\right)}{\mathcal{F}^{o}\left(n\left(\mathrm{H}_{2}\right), 3 N^{p}, T_{\text {kin }}\right)}
$$

The two panels of Fig. 15 show the relations between $\mathcal{R}$ and $n$ with $T_{\text {kin }}$ of $5 \mathrm{~K}, 14 \mathrm{~K}$ and $23 \mathrm{~K}$, and $N^{p}$ at $10^{11} \mathrm{~cm}^{-2}$ (low column density case, hereafter LN) and $10^{13} \mathrm{~cm}^{-2}$ (moderate column density case, hereafter $\mathrm{MN}$ ). In the $\mathrm{LN}$ case all transitions are optically thin and $\mathcal{R}$ increases monotonically with densities $n\left(\mathrm{H}_{2}\right) . \mathcal{R}$ is always larger than -0.45 in this case. In the MN case, the profiles of $\mathcal{R}$ along $n\left(\mathrm{H}_{2}\right)$ are similar to those in the LN case when $n\left(\mathrm{H}_{2}\right)>10^{5} \mathrm{~cm}^{-3}$. However, when the density is low $\left(n\left(\mathrm{H}_{2}\right) \sim 10^{3} \mathrm{~cm}^{-3}\right)$, the absorption line $2_{2,0}-2_{1,1}$ will be weak because the molecules are accumulated in lower energy states and the photons released from $2_{2,0}-1_{1,1}$ transition tend to be reabsorbed. For the low density $\left(n\left(\mathrm{H}_{2}\right) \sim 10^{3} \mathrm{~cm}^{-3}\right)$ and moderate column density $\left(N^{p}=10^{13} \mathrm{~cm}^{-2}\right)$ case, the $\mathcal{R}$ values are independent of kinetic temperature and always larger than -0.35 . Compared with the LN case, another characteristic of the MN case is that $\mathcal{R}$ will first slightly decrease as the density $n\left(\mathrm{H}_{2}\right)$ increases.

In the LN case with kinetic temperature fixed at $10 \mathrm{~K}$, the fitting between $\mathcal{R}$ and $n\left(\mathrm{H}_{2}\right)$ gives

$$
\log \left(n\left(\mathrm{H}_{2}\right)\right)=\frac{\ln (200 \mathcal{R}+80)}{1.45}+3
$$

For $\mathcal{R}$ smaller than -0.35 , the volume density should be smaller than or comparable with $10^{4}$ $\mathrm{cm}^{-3}$, but can not be well constrained. For $\mathcal{R}$ larger than -0.35 , the volume density should be larger 


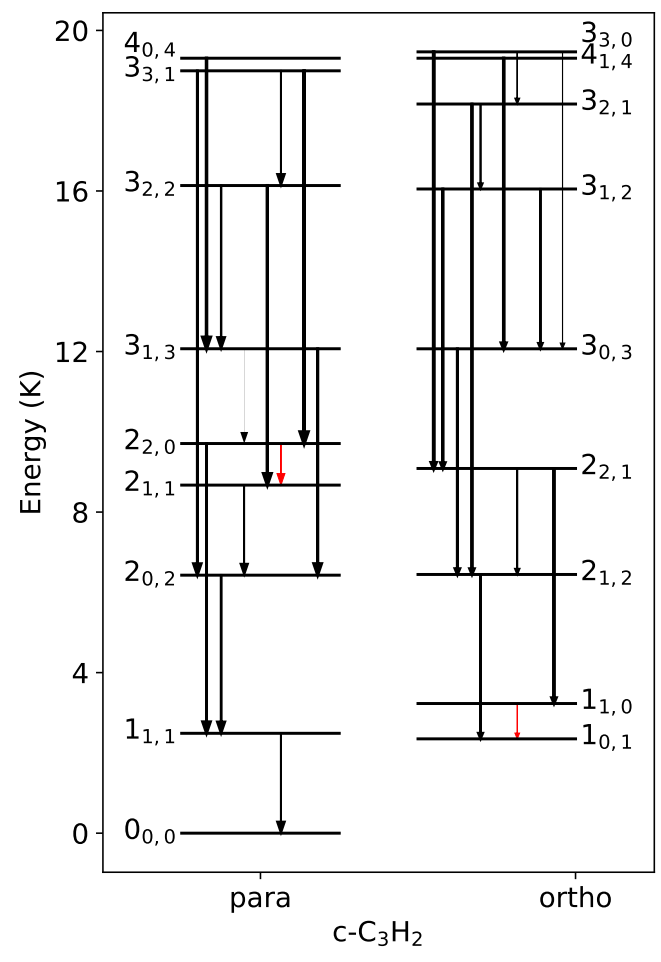

Figure 14. The transitions of $c-\mathrm{C}_{3} \mathrm{H}_{2}$. The widths of the arrows represent the corresponding Einstein coefficients for spontaneous transition. Red arrows: Observed transitions.

than $10^{4} \mathrm{~cm}^{-3}$. However, the assumption of the LN case may be not valid when the volume density is high, and Eq. D13 can only serve as a rough estimation of $n\left(\mathrm{H}_{2}\right)$.

If the kinetic temperature is fixed and $\mathcal{R}$ is not too low (larger than -0.35), the free parameters $n\left(\mathrm{H}_{2}\right)$ and $N^{p}$, as well as the optical depths can be directly fitted from Eq. D12.
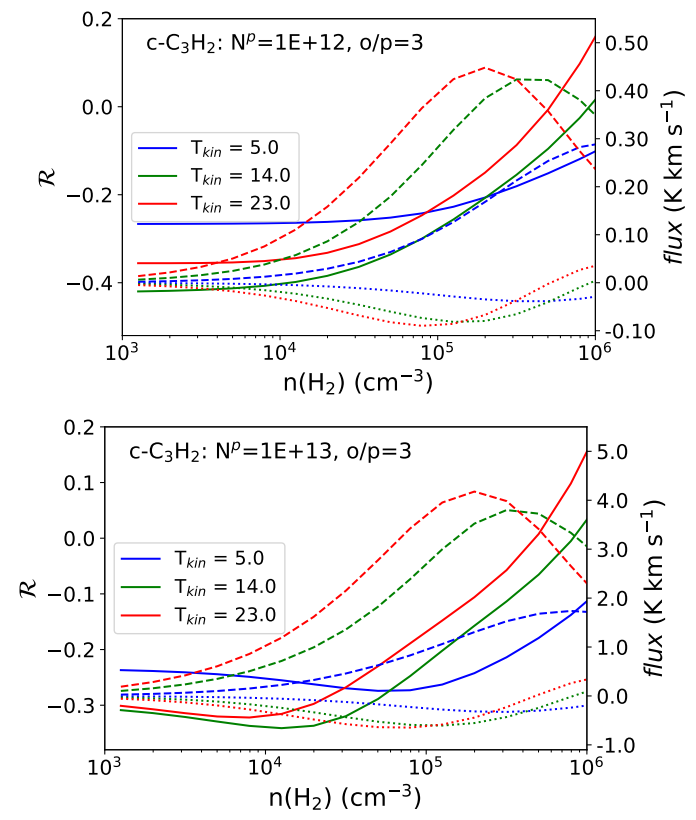

Figure 15. Upper panel: The dependence between $\mathcal{R}$ (flux ratio between $\mathrm{c}-\mathrm{C}_{3} \mathrm{H}_{2} 2_{2,0}-2_{1,1}$ and $\mathrm{c}-\mathrm{C}_{3} \mathrm{H}_{2} 1_{1,0^{-}}$ $\left.1_{0,1}\right)$ and $n\left(\mathrm{H}_{2}\right)$ with the column density of para- $\mathrm{C}_{3} \mathrm{H}_{2}$ $\left(N^{p}\right)$ fixed as $10^{12} \mathrm{~cm}^{-2}$. Lower panel: Same as the upper panel except with $N^{p}$ fixed as $10^{13} \mathrm{~cm}^{-2}$. The filled lines show the $\mathcal{R}$ values. The dashed and dotted lines show the fluxes of $\mathrm{c}-\mathrm{C}_{3} \mathrm{H}_{2} 1_{1,0}-1_{0,1}$ and $\mathrm{c}-\mathrm{C}_{3} \mathrm{H}_{2}$ $2_{2,0}-2_{1,1}$, respectively. 